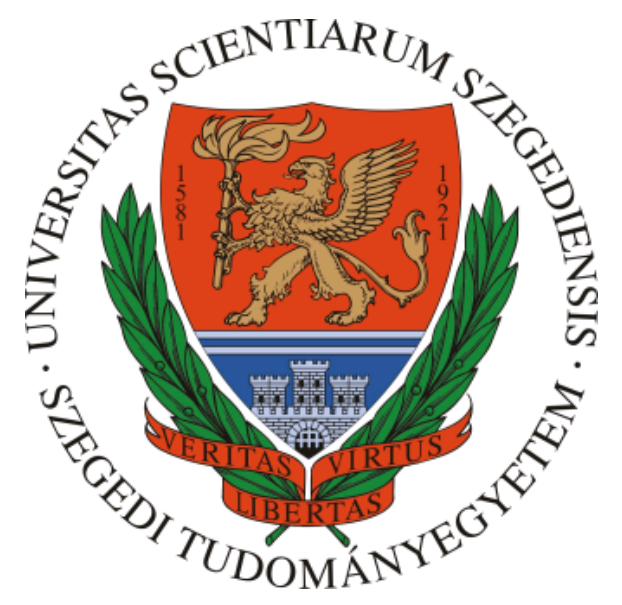

\title{
INDIVIDUALIZED TREATMENT OF EARLY BREAST CANCER
}

\author{
Ph.D. Thesis \\ Gyöngyi Kelemen, M.D. \\ Supervisor: \\ Prof. Zsuzsanna Kahán, M.D., Ph.D. \\ Department of Oncotherapy \\ Faculty of Medicine, University of Szeged \\ Szeged, Hungary
}

Szeged

2012 


\section{List of full papers that served as the basis of the Ph.D. thesis}

I. Pálka I, Kelemen G, Ormándi K, Lázár G, Nyári T, Thurzó L, Kahán Z

Tumour characteristics in screen-detected and symptomatic breast cancers

Pathology \& Oncology Research 14:(2) pp. 161-167. (2008)

IF: 1.260

II. Kelemen G, Farkas V, Debrah J, Ormándi K, Vörös A, Kaizer L, Varga Z, Lázár G, Kahán Z.

The relation of multifocality and tumour burden with various tumour characteristics and survival in early breast cancer

Neoplasma - submitted for publication

III. Kelemen G, Uhercsak G, Ormandi K, Eller J, Thurzo L, Kahan Z

Long-term efficiency and toxicity of adjuvant dose-dense sequential adriamycinpaclitaxel-cyclophosphamide chemotherapy in high-risk breast cancer Oncology 78:(3-4) pp. 271-273. (2010)

IF: 2.538

IV. Kelemen Gy, Varga Z, Lázár Gy, Thurzó L, Kahán Z

Cosmetic outcome 1-5 years after breast conservative surgery, irradiation and systemic therapy

Pathology \& Oncology Research - accepted for publication

IF: 1.483

\section{Related articles}

I. Varga Z, Cserhati A, Kelemen G, Boda K, Thurzo L, Kahan Z

The role of systemic therapy in the development of lung sequelae after conformal radiotherapy in breast cancer patients

International Journal of Radiation Oncology Physics 80:(4) pp. 1109-1116. (2011)

IF: 4.503 
II. Cserni G, Francz M, Kalman E, Kelemen G, Komjathy DC, Kovacs I, Kulka J, Sarkadi L, Udvarhelyi N, Vass L, Voros A

Estrogen receptor negative and progesterone receptor positive breast carcinomasHow frequent are they?

Pathology \& Oncology Research 17(3):663-8. (2011)

IF:1.483

III. Dobi A, Kelemen G, Kaizer L, Weiczner R, Thurzo L, Kahan Z

Breast cancer under 40 years of age: Increasing number and worse prognosis Pathology \& Oncology Research 17:(2) pp. 425-428. (2011)

IF: 1.483 
Table of contents

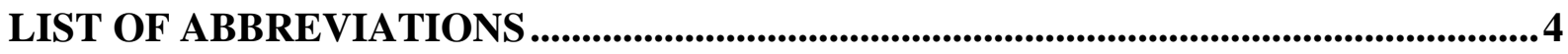

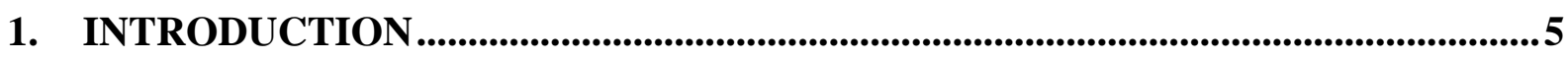

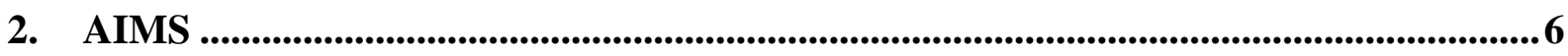

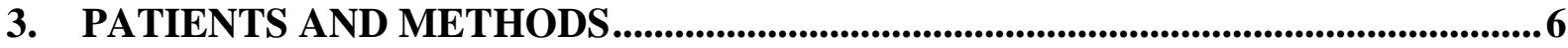

3.1 TUMOUR CHARACTERISTICS IN SCREEN-DETECTED AND SYMPTOMATIC BREAST CANCERS. .6

3.2 THE RELATION OF MULTIFOCALITY AND TUMOUR BURDEN WITH VARIOUS TUMOUR CHARACTERISTICS AND SURVIVAL IN EARLY BREAST CANCER .................................................. 7

3.3 THE EFFECT OF THE MAMMOGRAPHIC APPEARANCE ON SURVIVAL IN PATIENTS WITH HIGH

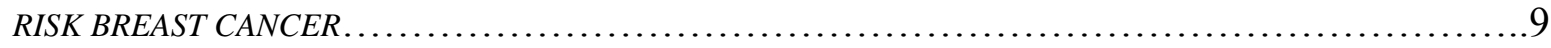

3.4 COSMETIC OUTCOME 1-5 YEARS AFTER BREAST CONSERVATIVE SURGERY, IRRADIATION AND SYSTEMIC THERAPY.

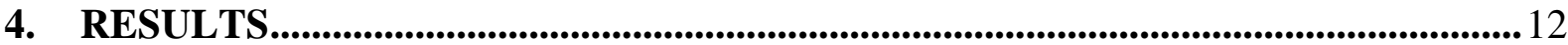

4.1 TUMOUR CHARACTERISTICS IN SCREEN-DETECTED AND SYMPTOMATIC BREAST CANCERS 12

4.2 THE RELATION OF MULTIFOCALITY AND TUMOUR BURDEN WITH VARIOUS TUMOUR

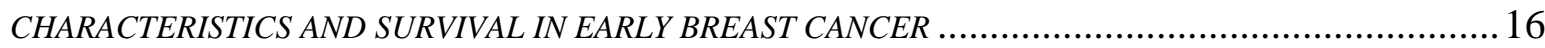

4.3 THE EFFECT OF THE MAMMOGRAPHIC APPEARANCE ON SURVIVAL IN PATIENTS WITH HIGH RISK BREAST CANCER

4.4 COSMETIC OUTCOME 1-5 YEARS AFTER BREAST CONSERVATIVE SURGERY, IRRADIATION AND SYSTEMIC THERAPY.

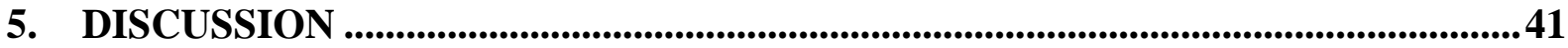

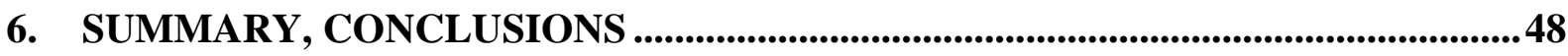

7. ACKNOWLEDGEMENTS............................................................................................................ 49

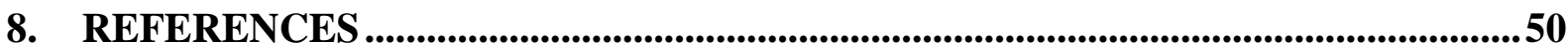

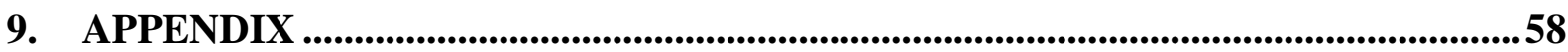




\section{List of abbreviations}

ABD axillary lymph node dissection

ATC adriamycin (A)-paclitaxel (T)-cyclophosphamide (C)

BCSS breast cancer-specific survival

BS breast separation

CMF cyclophosphamide, methotrexate and fluorouracil

DCIS ductal carcinoma in situ

DDFS distant disease-free survival

ER oestrogen receptor

FISH fluorescence in situ hybridization

HER2 human epidermal growth factor receptor 2

IMRT intensity-modulated radiation therapy

LVI lymphovascular invasion

OAR organ at risk

OS overall survival

pT size of the largest invasive tumour focus

PTV planning target volume

PR progesterone receptor

RFS relapse-free survival

SNB sentinel lymph node biopsy

TOP2A topoisomerase2-alpha 


\section{Introduction}

Breast carcinoma is a diverse disease entity, and the therapy should be based on specific markers reflecting its individual biological behaviour [1-3]. The currently used prognostic factors, however, do not reliably distinguish between true early breast cancers (usually screendetected and small, with a cure rate of around 95\%), and those that exhibit an apparently low TNM status, but in fact are at a more advanced stage with a high risk of relapse [3-6]. The role of mammographic service screening in the reduction of breast cancer mortality has been consistently revealed in numerous randomized controlled clinical studies and meta-analyses [7-11]. Thus, breast cancer-related mortality is significantly reduced in women invited to mammographic service screening as compared with those not invited to participate [7-11]. The type of mammographic image has recently been suggested as an independent prognostic factor. The presence of casting-type calcifications has been demonstrated to be a prognostic factor which carries a significantly higher risk of death as compared with cancers not associated with this mammographic abnormality [2, 12-16]. In contrast, stellate lesions on the mammogram reflect a more favourable prognosis than any other mammographic appearances $[2,16-17]$. The prognostic significance of multifocality/multicentricity and the tumour burden have long been the subjects of investigation, but the results are inconclusive as the nomenclature and methods applied were not uniform [1,18-23]. A larger tumour burden due to multifocality has been related to poorer pathological characteristics $[1,18,19]$, relapse-free survival (RFS) $[18,23]$ and overall survival (OS) $[18,21,22,24]$. In the case of multifocal breast cancers, therefore, a consideration of the TNM stage alone, would lead to inaccurate conclusions during treatment decision-making.

Breast-conserving surgery, usually followed by whole-breast irradiation, is the most widely used surgical option for early breast cancer [25-29]. The cosmetic and the functional outcome after postoperative breast radiotherapy depend on numerous patient- and therapyrelated factors. The radiogenic changes of the breast, such as dyspigmentation, teleangiectasia or breast oedema, fibrosis causing breast swelling and tenderness, depend on the dose, the irradiated volume and the individual radiosensitivity [30-33]. The impact of the systemic therapy on the cosmetic outcome has been the subject of numerous studies [34-38]. 


\section{Aims}

2.1 We set out to prospectively investigate the patient- and tumour-related features in early breast cancer shortly after the introduction of mammographic service-screening in Hungary.

2.2 In an extended database, we aimed at the evaluation of how the multifocality and calculated tumour burden in operable breast carcinomas relate to conventional pathological and other tumour features, and the assessment of their effects on the outcome.

2.3 The aim of a retrospective analysis of a clinical study with adjuvant dose-dense sequential adriamycin-paclitaxel-cyclophosphamide (ATC) chemotherapy was to investigate the impact of the breast cancers' mammographic appearance on survival in high risk breast cancer cases.

2.4 In a retrospective cohort analysis, we intended to study the patient- and therapy related factors that may influence the cosmetic and functional outcomes among our breast cancer patients after breast-conserving surgery and conformal radiotherapy, with or without adjuvant systemic therapy.

\section{Patients and methods}

\subsection{Tumour characteristics in screen-detected and symptomatic breast cancers}

Patients attending the Breast Unit of the University of Szeged, Hungary between May 1, 2004 and January 1, 2007 were eligible to take part in this study. 
The following data were prospectively registered: the age of the patient at the time of breast surgery, the type of breast surgery (breast-conserving surgery vs mastectomy), the type of lymph node surgery (sentinel lymph node biopsy (SNB) vs axillary lymph node dissection $(\mathrm{ABD})$ ), the pathological size of the largest invasive focus (pT), the histological type, the histological grade, the hormone receptor (oestrogen receptor (ER) and progesterone receptor (PR)) and human epidermal growth factor receptor 2 (HER2) status of the tumour and the presence of lymphovascular invasion (LVI). The mode of detection of the breast cancer was registered in the following categories: screen-detected (detected by breast imaging within the national mammography screening program or by opportunistic screening), symptomatic (detected via any symptom related to the tumour in a patient who did not attend any screening program in the last two years) or interval cancer (the tumour was diagnosed during the interval between two successive screening rounds and within 2 years after a negative screening finding). The mammographic appearance of the tumour, based on the mammography report, was registered. Mammographic images were classified according to Tabár et al. [12]. For the analysis of the association between the mammographic image and other characteristics of the tumour, the previous categories were grouped in the following way: spiculated lesions without calcifications, casting-type calcifications with or without an associated tumour mass, and others.

\section{Statistical analysis}

For the categorical parameters, chi-square or Fisher tests were applied; for the analysis of continuous data, variance analysis was used.

\subsection{The relation of multifocality and tumour burden with various tumour characteristics and survival in early breast cancer}

Women attending the Breast Unit at the University of Szeged with invasive breast cancer in clinical stage I or II between May 2004 and August 2010 were eligible for this study. All patients underwent primary breast surgery, and adjuvant therapy was administered in accordance with the national and international guidelines.

The data recorded were the age of the patient at the time of breast surgery, the mode of detection of the breast cancer (mammography screening-detected, detected other than by mammography screening or interval cancer) and its mammographic appearance. The 
radiologic images were categorized as stellate (spiculated) tumour masses, circular tumours, and parenchymal dystorsion/asymmetric density, while malignant microcalcifications were categorized into two groups: casting-type calcifications and non-casting-type calcifications. For the analysis of the association between the mammographic image and survival, these categories were grouped as: stellate lesions without casting-type calcifications, casting-type calcifications with or without an associated tumour mass and others. The type of breast surgery (breast-conserving surgery vs. mastectomy), the type of lymph node surgery (SNB vs. ABD with or without SNB), the pT, the histological type, the histological grade, the presence of LVI and the information on lymph node involvement were also compiled. The percentages of cells expressing the ER, the PR, Ki67 and topoisomerase2-alpha (TOP2A) protein were routinely determined by means of immunohistochemistry [39]. A cut-off value of $\geq 10 \%$ was used for ER or PR positivity, and $>15 \%$ for TOP2A or Ki67 positivity. The HER2 status was determined via immunohistochemistry and/or HER2 fluorescence in situ hybridization (FISH) [39]. Immunohistochemistry data were not available for all patients.

Additionally, we retrospectively extracted the following data from the pathological reports: the presence of multifocality, the sizes of the multiple foci (both invasive and in situ foci, if present), and the grade of the in situ component, if present. Pathological reports were considered only if there was a clear allusion to the presence or absence of more than one tumour focus. In all cases, large-format histological sections (maximum size 60x90 mm) were examined. The criterion of multifocality was the presence of more than one cancer focus separated by non-malignant breast tissue. If two or more invasive foci were present, the tumour was classified as invasive multifocal. Since most of the pathological reports did not provide the extent of the breast parenchyma involved by malignant structures, the pathological extent of the disease was estimated by summing the largest diameters of the invasive and in situ cancer foci; this measure was taken as the tumour burden. In unifocal cases, the tumour size comprised the tumour burden. Analyses were made on the basis of the presence of multifocality, the magnitude of the tumour burden, other pathological features and the survival data.

Survival data were collected on the basis of regular 6-month follow-up visits or events such as relapse or death. RFS was defined as the time from breast surgery to any instance of disease recurrence (local, regional or distant relapse or a contralateral breast cancer). Breast cancer- 
specific survival (BCSS) was defined as the time from breast surgery to death due to breast cancer. For the survival analyses, we excluded patients operated on after 2007. RFS and BCSS were studied in relation to the patient and tumour characteristics; the median value (19 $\mathrm{mm}$ ) of the tumour burden was applied as a cut-off value. In order to detect a difference in outcome between the apparently early (cancers $<15 \mathrm{~mm}$ ) and more advanced cases as a function of the studied variables, survival analysis was performed separately on a subgroup of patients with breast cancers measuring $<15 \mathrm{~mm}$, regardless of whether they were unifocal or multifocal.

\section{Statistical analyses}

For the categorical parameters, the chi-square test was applied; for the analysis of continuous data, variance analysis and the Kruskal-Wallis test were used. The effects of the different patient and pathological characteristics on the disease outcome were assessed with the Kaplan-Meier method, and the effects of the various tumour-related factors on the disease outcome were evaluated with the Cox proportional hazards model. Statistical analysis was performed with SPSS 15.0 for Windows.

\subsection{The effect of the mammographic appearance on survival in patients with high risk} breast cancer

Data of patients with high-risk breast cancer who received 4 cycles of adriamycin $60 \mathrm{mg} / \mathrm{m}^{2}$, paclitaxel $200 \mathrm{mg} / \mathrm{m}^{2}$ and cyclophosphamide $800 \mathrm{mg} / \mathrm{m}^{2}$ respectively, every 2 weeks were prospectively collected.

RFS and OS were defined as the time from enrolment to any disease recurrence (local, regional, distant relapse or contralateral breast cancer) or to death. After chemotherapy, during the first 5 years of follow-up, patients had regular checkups every 6 months including chest X-ray, abdominal ultrasound, blood tests, bilateral mammography and bone scan. Statistical analyses

Kaplan-Meier survival analysis and the Breslow test were used. 
3.4 Cosmetic outcome 1-5 years after breast conservative surgery, irradiation and systemic therapy

Eligible patients had undergone unilateral breast-conserving surgery, with or without SNB or/and ABD and conformal radiotherapy 1-5 years before the interview. Patients with prior malignancy or any other significant health problem were excluded, as were those on glucocorticoid therapy. The patients had been operated between May 2004 and December 2008, at either the Department of Surgery, University of Szeged, or at smaller surgical departments.

Use of the following adjuvant medical therapies was permitted: a taxane-based postoperative chemotherapy regimen (involving either docetaxel or paclitaxel at conventional doses) completed $\geq 4$ weeks prior to the radiotherapy $(n=23,13.1 \%$ ); adjuvant hormone therapy with either tamoxifen (20 mg/day) or an aromatase inhibitor (anastrozole, $1 \mathrm{mg} /$ day, or letrozole, $2.5 \mathrm{mg} /$ day), started $\geq 2$ weeks before the initiation of radiotherapy $(\mathrm{n}=49,24.7 \%$ and $\mathrm{n}=48$, $24.2 \%$, respectively); patients who did not receive any systemic medication during or after the radiotherapy were also eligible for enrolment $(n=75,37.9 \%)$.

CT-based three-dimensional treatment planning and conformal radiotherapy were performed in all cases with the patient in a supine position. All relevant technical details have been published previously [40]. Briefly, CT images were acquired at $1 \mathrm{~cm}$ intervals throughout the entire planning volume. The target volume and organs at risk (OARs) were contoured on the CT slices in the radiotherapy planning system. The planning target volume (PTV) coverage was analyzed via the dose-volume histograms and isodose visualization. Local or locoregional radiotherapy was chosen according to the local protocol. The tumour bed boost was delivered with either $6 \mathrm{MV}$ photon or 8-15 MeV electron fields. The radiation dose to the remaining breast parenchyma/chest wall and to the lymph nodes, if indicated, was $25 \times 2$ Gy (prescribed to the mean of the PTV); a tumour bed boost of 5-8x2 Gy was delivered when necessary. OAR constraints were used as previously described [40].

The following radiotherapy-related data were extracted from our database: the PTV, the volume of the PTV that received more than $47.5 \mathrm{~Gy}$, but less than $53.5 \mathrm{~Gy}$ (V95\%-107\%), the overdosed volume of the PTV (V>107\%), the volume and dose of the tumour bed boost, 
the technique used and the breast separation (BS), i.e. the distance between the points at which the tangential fields entered the body.

The cosmetic outcome was evaluated at a single routine 6-month check-up visit, 1-5 years after the radiotherapy. The patients were examined, and a questionnaire relating to the following items was completed: the overall cosmetic success in the opinions of the patient and the physician (G. K. or Z. K.), the presence of breast fibrosis or oedema, teleangiectasia or dyspigmentation, all scored on a 4-point categorical scale according to the modified system of Johansen et al. [36, 41]. Briefly, the following scoring system was used. Breast fibrosis: 0: none, 1: density slightly increased, 2 : increased density and firmness, 3 : very marked density with retraction; Oedema: 0: none, 1: trace thickening of the skin, 2: marked oedema, leathery skin texture, 3: severe oedema with papillary formation; Teleangiectasia: 0 : none, $1:<1 \mathrm{~cm}$, 2: 1-4 cm, 3: >4 cm; Dyspigmentation: 0: none, 1: mild, 2: moderate, 3: severe. Physicians classified the cosmetic result as excellent if no asymmetry or changes of the skin or the breast contour occurred; in the case of slight, moderate or severe manifestation of at least one of these factors, the outcome was considered good, fair or poor, respectively. The patients were asked whether they felt pain or tenderness in the operated breast, and whether they had experienced changes in their body image or in their clothing habits. The length of the excision scar and the difference (regarded as measurable when $\geq 1.0 \mathrm{~cm}$ ) in the jugulum-nipple distance (indicative of breast asymmetry) were recorded. Data were additionally collected on smoking habits, with the participants categorized as past or present smokers or non-smokers.

\section{Statistical analyses}

The various patient- and radiotherapy-related characteristics associated with the cosmetic and functional outcomes were analyzed by the means of the chi-square test, analysis of variance and logistic regression. The Kappa test was applied to investigate the connection between the opinions of the physicians and the patients as concerns the cosmetic outcome. Binary univariate logistic regression models were first utilized separately, followed by the multivariate logistic regression model to examine joint effects and interactions. Statistical analysis was performed with SPSS 15.0 for Windows. 


\section{Results}

\subsection{Tumour characteristics in screen-detected and symptomatic breast cancers}

The data on 565 patients with 569 invasive breast cancers were collected. (Four patients had synchronous bilateral invasive breast cancer.)

Patient- and tumour-related characteristics according to the mode of detection

Overall, 258 tumours (46\%) were screen-detected, while 263 (46\%) were symptomatic and $48(8 \%)$ were interval cancers. The mean \pm SD age of the overall patient population at the time of breast surgery was $58.1 \pm 10.9$ years (range 27.8-85.1), while that of the cases with screen-detected or symptomatic tumours was $58.4 \pm 7.6$ and $58.5 \pm 13.9$ years, respectively, and that of the patients with interval cancers was $54.3 \pm 5.4$ years $(\mathrm{p}=0.04)$. While $35.4 \%$ of the patients with interval cancer, and $31 \%$ of the patients with symptomatic cancer were premenopausal, only $20.2 \%$ of the patients with screen-detected cancer were premenopausal $(\mathrm{p}=0.007)$. The pathological tumour characteristics are presented in Table 1 .

Surgical and medical treatment options according to the mode of detection

The rate of breast-conserving surgery among the patients with screen-detected cancers was significantly $(\mathrm{p}<0.001)$ higher than that in symptomatic or interval cancer cases. Similarly, the rate of SNB was the highest in the group with screen-detected tumours ( $\mathrm{p}<0.001)$. In 15 clinically node-negative cases, no axillary surgery was performed because of the advanced age of the patient. Adjuvant chemotherapy was significantly less frequently applied in the patients with screen-detected cancers $(36.8 \%)$ than in the symptomatic $(53.6 \%)$ or interval cancer $(66.7 \%)$ cases $(\mathrm{p}<0.001)$. The frequency of use of hormone therapy was similar in the three groups (Table 2).

Patient- and tumour-related characteristics according to the mammographic image

In two cases, no mammography had been performed prior to surgery, and in another three cases, the result of the mammography was not available. Different characteristics according to the mammographic image are presented in table 3 . The cancers associated with castingtype calcifications on the mammogram were significantly more often of ductal type $(\mathrm{p}=0.043$, Fisher's exact test), of grade 3 ( $p<0.001)$, ER- and PR-negative $(p<0.001)$ and HER2-positive $(\mathrm{p}<0.001)$ than the cancers without casting calcifications. The mammographic images revealed no differences in tumour size, lymph node status or LVI. 
Screen-detected Symptomatic (\%) Interval cancer p

(\%)

(\%)

\begin{tabular}{|c|c|c|c|c|}
\hline \multicolumn{5}{|l|}{ Histological type } \\
\hline $\begin{array}{l}\text { Invasive ductal } \\
\text { carcinoma }\end{array}$ & $201 / 258(78.2)$ & $203 / 263(77.2)$ & $31 / 48(64.6)$ & 0.168 (chi-square test) \\
\hline $\begin{array}{l}\text { Invasive lobular } \\
\text { carcinoma }\end{array}$ & $35 / 258(13.6)$ & $38 / 263(14.4)$ & 7/48 (14.6) & 0.21 (Fisher's exact test) \\
\hline $\begin{array}{l}\text { Others } \\
\text { (medullary, } \\
\text { mucinous, } \\
\text { tubular, } \\
\text { papillary) }\end{array}$ & $22 / 258(8.2)$ & $22 / 263(8.4)$ & $10 / 48(20.8)$ & \\
\hline \multicolumn{5}{|l|}{ Grade } \\
\hline 1 & $75 / 257(29.2)$ & $26 / 260(10.0)$ & $8 / 48(16.7)$ & $<0.001$ (chi-square test) \\
\hline 2 & $115 / 257(44.7)$ & $124 / 260(47.7)$ & $22 / 48(45.8)$ & \\
\hline 3 & $67 / 257(26.1)$ & $110 / 260(42.3)$ & $18 / 48(37.5)$ & \\
\hline \multicolumn{5}{|l|}{ Tumour size } \\
\hline $1-10 \mathrm{~mm}$ & $88 / 258(34.1)$ & $19 / 263(7.2)$ & $3 / 48(6.2)$ & $<0.001$ (chi-square test) \\
\hline $11-20 \mathrm{~mm}$ & $120 / 258(46.5)$ & $93 / 263(35.4)$ & $24 / 48(50)$ & \\
\hline$>20 \mathrm{~mm}$ & $50 / 258(19.4)$ & $151 / 263(57.4)$ & $21 / 48(43.8)$ & \\
\hline \multicolumn{5}{|l|}{ Node } \\
\hline Negative & $179 / 258(69.4)$ & $127 / 263(48.3)$ & $24 / 48(50)$ & $<0.001$ (chi-square test) \\
\hline Positive & $79 / 258(30.6)$ & $136 / 263(51.7)$ & $24 / 48(50)$ & \\
\hline \multicolumn{5}{|l|}{ LVI } \\
\hline Negative & $217 / 258(84.1)$ & $186 / 263(70.7)$ & $37 / 48(77.1)$ & 0.001 (chi-square test) \\
\hline Positive & 41/258 (15.9) & $77 / 263(29.3)$ & $11 / 48(22.9)$ & \\
\hline \multicolumn{5}{|l|}{ ER and PR } \\
\hline Positive & $218 / 257(84.8)$ & 208/262 (79.4) & $37 / 48(77.1)$ & 0.193 (chi square test) \\
\hline Negative & $39 / 257(15.2)$ & $54 / 262(20.6)$ & $11 / 48(22.9)$ & \\
\hline \multicolumn{5}{|l|}{ HER2 } \\
\hline Negative & $222 / 255(87.1)$ & $220 / 262(84.0)$ & $38 / 48(79.2)$ & 0.310 (chi-square test) \\
\hline Positive & 33/255 (12.9) & $42 / 262(16.0)$ & $10 / 48(20.8)$ & \\
\hline
\end{tabular}

Table 1 Pathological tumour characteristics for the various modes of detection 


\begin{tabular}{|c|c|c|c|c|}
\hline & $\begin{array}{l}\begin{array}{l}\text { Screen- } \\
\text { detected } \\
\mathrm{n}=258(\%)\end{array} \\
\end{array}$ & $\begin{array}{l}\text { Symptomatic } \\
\mathrm{n}=263(\%)\end{array}$ & $\begin{array}{l}\text { Interval cancer } \\
\mathrm{n}=48(\%)\end{array}$ & $\begin{array}{l}\mathbf{p} \text { (chi-square } \\
\text { test) }\end{array}$ \\
\hline \multicolumn{5}{|l|}{ Breast surgery } \\
\hline $\begin{array}{l}\text { Breast-conserving } \\
\text { surgery }\end{array}$ & $223(86.4)$ & $131(49.8)$ & $26(54.2)$ & $<0.001$ \\
\hline Mastectomy & $35(13.6)$ & $132(50.2)$ & $22(45.8)$ & \\
\hline \multicolumn{5}{|l|}{ Lymph node surgery } \\
\hline $\begin{array}{l}\text { Sentinel node } \\
\text { biopsy }\end{array}$ & $116(45.0)$ & $43(16.3)$ & $10(20.8)$ & $<0.001$ \\
\hline $\begin{array}{l}\text { Axillary lymph node } \\
\text { dissection } \pm \text { sentinel } \\
\text { biopsy }\end{array}$ & $137(53.1)$ & $210(79.8)$ & $38(79.2)$ & \\
\hline $\begin{array}{l}\text { No axillary } \\
\text { surgery }\end{array}$ & $5(1.9)$ & $10(3.9)$ & $0(0)$ & \\
\hline \multicolumn{5}{|l|}{$\begin{array}{l}\text { Adjuvant } \\
\text { chemotherapy }\end{array}$} \\
\hline No & $163(63.2)$ & $122(46.4)$ & $16(33.3)$ & $<0.001$ \\
\hline Yes & $95(36.8)$ & $141(53.6)$ & $32(66.7)$ & \\
\hline \multicolumn{5}{|l|}{$\begin{array}{l}\text { Adjuvant hormone } \\
\text { therapy }\end{array}$} \\
\hline No & $93(36.1)$ & $80(30.4)$ & $16(33.3)$ & 0.395 \\
\hline Yes & $165(63.9)$ & $183(69.6)$ & $32(66.7)$ & \\
\hline
\end{tabular}

Table 2 Surgical and medical therapy following the various modes of detection of breast cancer 


\begin{tabular}{|c|c|c|c|c|}
\hline & $\begin{array}{c}\text { Stellate lesions } \\
\text { without } \\
\text { calcifications } \\
(\%)\end{array}$ & $\begin{array}{c}\text { Casting-type } \\
\text { calcifications } \pm \\
\text { associated } \\
\text { tumour mass } \\
(\%)\end{array}$ & Others (\%) & $\mathbf{p}$ \\
\hline \multicolumn{5}{|l|}{$\begin{array}{l}\text { Histological } \\
\text { type }\end{array}$} \\
\hline $\begin{array}{l}\text { Invasive } \\
\text { ductal cancer }\end{array}$ & $165 / 213(77.5)$ & $38 / 40(95)$ & $229 / 311(73.5)$ & 0.056 (chi-square test) \\
\hline $\begin{array}{l}\text { Invasive } \\
\text { lobular cancer }\end{array}$ & 29/213 (13.6) & $1 / 40(2.5)$ & 48/311 (15.4) & $\begin{array}{l}0.046 \text { (Fisher's exact } \\
\text { test) }\end{array}$ \\
\hline $\begin{array}{l}\text { Others } \\
\text { (medullary, } \\
\text { mucinous, } \\
\text { tubular, } \\
\text { papillary) }\end{array}$ & $19 / 213(8.9)$ & $1 / 40(2.5)$ & $34 / 311(10.1)$ & \\
\hline \multicolumn{5}{|l|}{ Grade } \\
\hline 1 & $55 / 212(25.9)$ & $2 / 40(5.0)$ & $52 / 308(16.9)$ & $<0.001$ (chi-square test) \\
\hline 2 & $110 / 212(51.9)$ & $11 / 40(27.5)$ & $136 / 308(44.2)$ & \\
\hline 3 & $47 / 212(22.2)$ & $27 / 40(67.5)$ & $120 / 308(38.9)$ & \\
\hline \multicolumn{5}{|l|}{ Tumour size } \\
\hline $1-10 \mathrm{~mm}$ & $42 / 213(19.7)$ & $8 / 40(20.0)$ & 59/311 (19.0) & 0.098 (chi-square test) \\
\hline $11-20 \mathrm{~mm}$ & $100 / 213(47.0)$ & $11 / 40(27.5)$ & $123 / 311(39.5)$ & \\
\hline$>20 \mathrm{~mm}$ & $71 / 213(33.3)$ & $21 / 40(52.5)$ & $129 / 311(41.5)$ & \\
\hline \multicolumn{5}{|l|}{ Node } \\
\hline Negative & $123 / 213(57.7)$ & $19 / 40(47.5)$ & $185 / 311(59.5)$ & 0.350 (chi-square test) \\
\hline Positive & $90 / 213(42.3)$ & $21 / 40(52.5)$ & $126 / 311(40.5)$ & \\
\hline \multicolumn{5}{|l|}{ LVI } \\
\hline Negative & $168 / 213(78.9)$ & $27 / 40(67.5)$ & $241 / 311(77.5)$ & 0.287 (chi-square test) \\
\hline Positive & 45/213 (21.1) & $13 / 40(32.5)$ & 70/311 (22.5) & \\
\hline \multicolumn{5}{|l|}{ ER and PR } \\
\hline Positive & $189 / 212(89.1)$ & $23 / 40(42.5)$ & $247 / 310(79.7)$ & $<0.001$ (chi-square test) \\
\hline Negative & $23 / 212(10.9)$ & $17 / 40(57.5)$ & $63 / 310(20.3)$ & \\
\hline \multicolumn{5}{|l|}{ HER2 } \\
\hline Negative & $198 / 212(93.4)$ & $18 / 40(45.0)$ & $259 / 308(84.1)$ & $<0.001$ (chi-square test) \\
\hline Positive & $14 / 212(6.6)$ & $22 / 40(55.0)$ & 49/308 (15.9) & \\
\hline
\end{tabular}

Table 3 Comparison of the pathological tumour characteristics with the mammographic image 
4.2 The relation of multifocality and tumour burden with various tumour characteristics and survival in early breast cancer

Among a total of 1234 breast carcinoma cases, 1071 were eligible for the analysis. The mean \pm SD age was $58.6 \pm 12.0$ (range $24.5-88.6$ ) years. The patient and tumour characteristics are presented in Tables 4 and 5. Around $40 \%$ of the cases were screen-detected. Among the $796(74.3 \%)$ unifocal and $275(25.7 \%)$ multifocal cancers found, there were 101 multifocal invasive tumours, while in 174 cases a single invasive focus was associated with one or more in situ foci.

\section{The connection of multifocality with different tumour features}

Multifocal cancers were more susceptible to screen detection, HER2 positivity and casting calcifications in the mammogram than were unifocal cancers. Invasive multifocality was more strongly associated than non-invasive multifocality with mastectomy (44\% vs. $28 \%, \mathrm{p}<0.01$ ), lymph node positivity (47 vs. $35 \%, \mathrm{p}=0.03$ ) and HER 2 positivity (17 vs. 9\%, p=0.02). The maximum diameter of unifocal cancers was greater than the largest tumour focus in multifocal cancers $(\mathrm{p}<0.001)$.

The connection between the tumour burden and different patient and tumour characteristics

The calculated mean \pm SE tumour burden in the unifocal and the multifocal cases was $19.5 \pm 0.4$ and $31.2 \pm 0.9$, respectively $(\mathrm{p}<0.001)$. We analyzed the standard pathological parameters according to the tumour burden, using the median value of $19.0 \mathrm{~mm}$ as a threshold (Table 6). The presence of lymph node metastases $(\mathrm{p}<0.001)$ or LVI $(\mathrm{p}<0.001)$ was associated with a larger tumour burden. The mean $\pm \mathrm{SE}$ tumour burden was $21.7 \pm 0.4$ vs. $28.9 \pm 1.4 \mathrm{~mm}$ in invasive ductal vs. lobular carcinomas, respectively $(\mathrm{p}<0.01)$. A larger invasive tumour focus and a larger tumour burden predisposed to ER, PR negativity $(\mathrm{p}<0.001)$ and HER2 positivity ( $\mathrm{p}<0.001)$. In multivariate analysis, only the connections between the tumour burden and ER, PR negativity and HER2 positivity remained significant $(\mathrm{p}<0.001)$. A larger tumour burden was associated with Ki67 positivity $(\mathrm{p}=0.02)$. A tumour burden larger than the cut-off value was related to multifocality $(\mathrm{p}<0.001)$. The mean \pm SE tumour burden was $32.6 \pm 0.2 \mathrm{~mm}$ in cases where there were casting calcifications in the mammogram, and $20.9 \pm 0.6 \mathrm{~mm}$ when the lesion was categorized as a spiculated mass $(\mathrm{p}<0.001)$. 
Variable

Symptomatic+interval

Mammographic Stellate

appearance

Age (mean \pm SD)

Mode of

Screen-detected

detection

Casting-type

Calcification \pm tumour mass

Other

$\begin{array}{ll}\text { Breast surgery } & \text { Excision } \\ & \text { Mastectomy }\end{array}$

Lymph node surgery

Sentinel lymph node biopsy or nothing

Axillary lymph node dissection \pm sentinel lymph node biopsy

$\begin{array}{llll}\begin{array}{l}\text { Unifocal } \\ \text { cancers } \\ (\mathrm{n}=796)\end{array} & \begin{array}{l}\text { Multifocal } \\ \text { cancers } \\ (\mathrm{n}=275)\end{array} & \mathbf{p} & \text { All } \\ (\%) & (\%) & (\mathrm{n}=1071) \\ & & (\%)\end{array}$

$58.7 \pm 12.2 \quad 58.5 \pm 11.5$

0.87

$58.6 \pm 12.0$

$312(39.2) \quad 130(47.3) \quad 0.02$

$442(41.3)$

$484(60.8) \quad 145(52.7)$

$629(58.7)$

$308(39.1) \quad 78(28.4)$

$<0.001$

$386(36.3)$

$35(4.4) \quad 51(18.5)$

$86(8.1)$

$444(56.4) \quad 146(53.1)$

$590(55.6)$

$570(71.6) \quad 186(67.6) \quad 0.22 \quad 756(70.6)$

$226(28.4) \quad 89(32.4)$

$315(29.4)$

$382(48.0) \quad 139(50.5) \quad 0.48$

382 (48.6)

$414(52.0) \quad 136(49.5)$

414 (51.4)

Table 4 Patient-, tumour- and surgery-related parameters in unifocal and multifocal cancers 


\begin{tabular}{|c|c|c|c|c|c|c|c|c|}
\hline \multicolumn{2}{|l|}{ Variable } & \multirow{2}{*}{$\begin{array}{l}\begin{array}{l}\text { Unifocal } \\
\text { cancers } \\
(\mathrm{n}=796)(\%)\end{array} \\
19.5 \pm 10.8\end{array}$} & \multirow{2}{*}{$\begin{array}{l}\begin{array}{l}\text { Multifocal } \\
\text { cancers } \\
(\mathrm{n}=275)(\%)\end{array} \\
16.3 \pm 9.6\end{array}$} & \multirow{2}{*}{$\begin{array}{l}\mathbf{p} \\
\text { (multifocal } \\
\text { versus } \\
\text { unifocal) } \\
\\
<0.001\end{array}$} & \multirow{2}{*}{$\begin{array}{l}\text { All } \\
(\mathrm{n}=1071) \\
(\%) \\
18.6 \pm 10.6\end{array}$} & \multirow{2}{*}{$\begin{array}{l}\text { Invasive } \\
\text { multifocal } \\
\text { cancers } \\
(\mathrm{n}=101)(\%)\end{array}$} & \multirow{2}{*}{$\begin{array}{l}\mathbf{p} \\
\text { (invasive } \\
\text { multifocal } \\
\text { versus } \\
\text { unifocal) } \\
0.24\end{array}$} & \multirow{2}{*}{$\begin{array}{l}\text { All } \\
(\mathrm{n}=897) \\
(\%) \\
19.3 \pm 10.7\end{array}$} \\
\hline $\begin{array}{l}\text { Tumour size } \\
(\text { mean } \pm \text { SD })\end{array}$ & & & & & & & & \\
\hline $\begin{array}{l}\text { Lymph node } \\
\text { status }\end{array}$ & positive & $286(35.9)$ & $102(37.1)$ & & $388(36.2)$ & $47(46.5)$ & & $333(37.1)$ \\
\hline \multirow{2}{*}{$\begin{array}{l}\text { Histological } \\
\text { type of the } \\
\text { invasive } \\
\text { component }\end{array}$} & $\begin{array}{l}\text { Invasive ductal } \\
\text { carcinoma }\end{array}$ & $620(77.9)$ & $220(80.0)$ & 0.61 & $840(78.4)$ & 77 (76.2) & 0.10 & 697 (77.7) \\
\hline & & $83(10.4)$ & $23(8.4)$ & & $106(9.9)$ & $6(5.9)$ & & 89 (9.9) \\
\hline \multirow[t]{3}{*}{ Grade } & & & & & $n=1062$ & & & $\mathrm{n}=888$ \\
\hline & 1 & $113(14.3)$ & $45(16.5)$ & 0.38 & $158(14.9)$ & $16(16.2)$ & 0.65 & $129(14.5)$ \\
\hline & 2 or 3 & $676(85.7)$ & $228(83.5)$ & & $904(85.1)$ & $83(83.8)$ & & $759(85.5)$ \\
\hline Presence of & & $\mathrm{n}=295$ & $\mathrm{n}=197$ & 0.49 & $n=492$ & $\mathrm{n}=58$ & & $\mathrm{n}=353$ \\
\hline
\end{tabular}




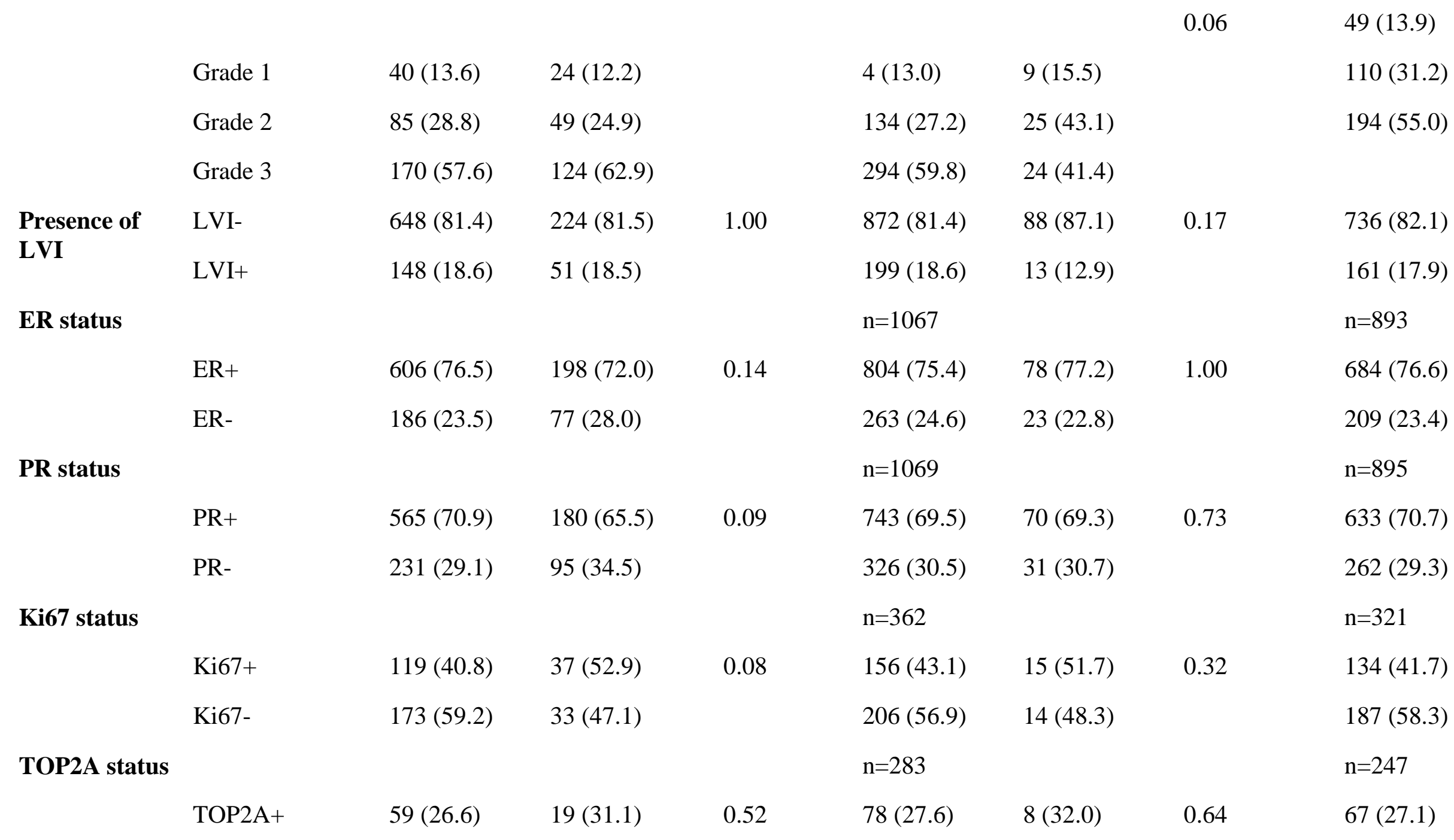




\begin{tabular}{|c|c|c|c|c|c|c|c|c|}
\hline \multirow{3}{*}{ HER2 status } & TOP2A- & 163 (73.4) & $42(68.9)$ & & $205(72.4)$ & $17(68.0)$ & & $180(72.9)$ \\
\hline & & & & & $\mathrm{n}=1048$ & & \multirow{3}{*}{0.003} & $\mathrm{n}=879$ \\
\hline & HER2+ & $58(7.4)$ & $46(17.2)$ & $<0.001$ & $104(9.9)$ & $17(17.3)$ & & $75(8.5)$ \\
\hline \multirow{4}{*}{$\begin{array}{l}\text { Triple } \\
\text { negativity }\end{array}$} & HER2- & 723 (92.6) & $221(82.8)$ & & $944(90.1)$ & $81(82.7)$ & & 804 (91.5) \\
\hline & & & & & $\mathrm{n}=1063$ & & \multirow{3}{*}{0.08} & $\mathrm{n}=890$ \\
\hline & Yes & $106(13.4)$ & $26(9.5)$ & 0.09 & $132(12.4)$ & $7(6.9)$ & & $113(12.7)$ \\
\hline & No & 683 (86.6) & $248(90.5)$ & & 931 (87.6) & $94(93.1)$ & & 777 (87.3) \\
\hline
\end{tabular}

Table 5 Routinely assessed pathological parameters among unifocal, multifocal and invasive multifocal 


\begin{tabular}{|c|c|c|c|c|}
\hline \multicolumn{2}{|l|}{ Variable } & \multirow{2}{*}{$\begin{array}{l}\text { Tumour burden } \\
\leq 19 \text { mm }(\mathrm{n}=536) \\
12.4 \pm 3.9\end{array}$} & \multirow{2}{*}{$\begin{array}{l}\text { Tumour burden } \\
>\mathbf{1 9} \mathbf{~ m m}(\mathrm{n}=535) \\
24.9 \pm 11.4\end{array}$} & \multirow{2}{*}{$\begin{array}{l}\mathbf{p} \\
<0.001\end{array}$} \\
\hline $\begin{array}{l}\text { Tumour size } \\
(\text { mean } \pm \text { SD })\end{array}$ & & & & \\
\hline \multirow{2}{*}{$\begin{array}{l}\text { Lymph node } \\
\text { status }\end{array}$} & negative & $383(71.5)$ & $300(56.1)$ & $<0.001$ \\
\hline & positive & $153(28.5)$ & 235 (43.9) & \\
\hline \multirow[t]{4}{*}{$\begin{array}{l}\text { Histological } \\
\text { type }\end{array}$} & $\begin{array}{l}\text { Invasive ductal } \\
\text { carcinoma }\end{array}$ & $437(81.5)$ & 403 (75.3) & 0.01 \\
\hline & $\begin{array}{l}\text { Invasive lobular } \\
\text { carcinoma }\end{array}$ & $46(8.6)$ & 79 (14.8) & \\
\hline & Other & & & \\
\hline & & $53(9.9)$ & $53(9.9)$ & \\
\hline \multirow[t]{2}{*}{ Grade } & 1 & $118(22.2)$ & $40(7.5)$ & $<0.001$ \\
\hline & 2 or 3 & $411(77.8)$ & $490(92.5)$ & \\
\hline \multirow[t]{4}{*}{$\begin{array}{l}\text { Presence of } \\
\text { DCIS }\end{array}$} & Grade 1 & $\begin{array}{l}n=219(44.5 \% \text { of } \\
\text { all })\end{array}$ & $\begin{array}{l}n=273(55.5 \% \text { of } \\
\text { all })\end{array}$ & $<0.001$ \\
\hline & Grade? & $44(20.1)$ & $20(7.3)$ & \\
\hline & Grade 3 & $69(31.5)$ & $65(23.8)$ & \\
\hline & & $106(48.4)$ & 188 (68.9) & \\
\hline \multirow{2}{*}{$\begin{array}{l}\text { Presence of } \\
\text { LVI }\end{array}$} & LVI- & $460(85.8)$ & $412(77.0)$ & $<0.001$ \\
\hline & LVI+ & $76(14.2)$ & $123(23.0)$ & \\
\hline ER status & ER+ & $436(82.0)$ & $368(68.8)$ & $<0.001$ \\
\hline$n=1067$ & ER- & $96(31.2)$ & 167 (31.2) & \\
\hline PR status & $\mathrm{PR}+$ & $403(75.5)$ & 340 (63.6) & $<0.001$ \\
\hline$n=1069$ & PR- & $131(24.5)$ & 195 (36.4) & \\
\hline Ki67 status & Ki67+ & $76(37.4)$ & $80(50.3)$ & 0.02 \\
\hline$n=362$ & Ki67- & $127(62.6)$ & 79 (49.7) & \\
\hline
\end{tabular}




$\begin{array}{lllll}\text { TOP2A status } & \text { TOP2A+ } & 38(24.7) & 40(31.0) & 0.29 \\ \mathrm{n}=283 & \text { TOP2A- } & 116(75.3) & 89(69.0) & \\ \begin{array}{l}\text { HER2 status } \\ \mathrm{n}=1048\end{array} & \text { HER2+ } & 39(7.4) & 65(12.4) & 0.01 \\ \text { Multifocality } & \text { Yes } & 486(92.6) & 458(87.6) & \\ \mathrm{n}=1071 & \text { No } & 60(11.2) & 215(40.2) & <0.001 \\ & & 476(88.8) & 320(59.8) & \end{array}$

Table 6 Routinely assessed pathological parameters in 1071 breast cancers according to tumour burden 


\section{Survival data}

The median follow-up time for the population of 584 patients participating in the survival analysis was 5.0 (range 0.3-7.3) years. There were 65 relapses (12.7\%) and 30 deaths $(5.8 \%)$. The numbers of relapses and deaths in the multifocal vs. the unifocal cases were 17 vs. 48 and 7 vs. 23, respectively. The survival data did not differ as a function of the presence of multifocality in the population. Among the conventional tumour characteristics, a larger invasive tumour, the presence of LVI or lymph node involvement, HER2 positivity and triple negativity were associated with a poorer RFS and OS (Table 7). The grade of the invasive tumour was not related to the RFS. As regards the mode of detection, screen-detected tumours gave RFS and OS statistics that were superior to those for non-screen-detected or interval cancers. The mammographic appearance of the tumour was not related to the outcome. A tumour burden $>19 \mathrm{~mm}$ or $>40 \mathrm{~mm}$ (extensive tumour) involved a shorter RFS and OS. Neither multifocality nor invasive multifocality was associated with a shorter RFS or OS.

In Cox proportional hazards models, the pT, the lymph node status, triple negativity and HER2 positivity remained independent determinants of an increased risk of relapse or death (Tables 8 and 9). 


\section{\begin{tabular}{ll}
\hline RFS &
\end{tabular} \\ OS \\ estimated mean $( \pm \mathrm{SE}$, years $) \quad$ estimated mean $( \pm \mathrm{SE}$, years $)$}

\section{Largest invasive tumour}

$\begin{array}{ccc}<15 \mathrm{~mm} & 7.0 \pm 0.1 & 7.3 \pm 0.0 \\ \geq 15 \mathrm{~mm} & 6.4 \pm 0.1 & 6.9 \pm 0.1 \\ p \text { (Mantel-Cox) } & <0.001 & <0.001\end{array}$

Lymph node positivity

$\begin{array}{ccc}\text { Yes } & 6.1 \pm 0.2 & 6.8 \pm 0.1 \\ \text { No } & 7.0 \pm 0.1 & 7.2 \pm 0.0 \\ p \text { (Mantel-Cox }) & <0.001 & <0.001\end{array}$

Invasive tumour grade

$\begin{array}{lcc}1 & 6.9 \pm 0.2 & 7.3 * \\ 2 \text { or } 3 & 6.6 \pm 0.1 & 6.9 \pm 0.6 \\ p(\text { Mantel-Cox }) & =0.17 & =0.03\end{array}$

\section{Presence of LVI}

$\begin{array}{ccc}\text { Yes } & 6.2 \pm 0.2 & 6.7 \pm 0.1 \\ \text { No } & 6.8 \pm 0.1 & 7.2 \pm 0.1 \\ p \text { (Mantel-Cox }) & =0.02 & =0.004\end{array}$

\section{HER2}

$\begin{array}{ccc}\text { Positive } & 5.7 \pm 0.4 & 6.3 \pm 0.2 \\ \text { Negative } & 6.7 \pm 0.1 & 7.1 \pm 0.1 \\ p \text { (Mantel-Cox }) & <0.001 & <0.001\end{array}$

Triple negative

Yes

$6.0 \pm 0.3$

$6.5 \pm 0.2$ 
No

$p$ (Mantel-Cox)

Mode of detection

Screen-detected

Interval and non-

screen-detected cancer

p (Mantel-Cox)

Mammographic

appearance

Spiculated tumour mass without casting calcification

Casting calcification \pm tumour mass

Other

$p$ (Mantel-Cox)

Multifocality

Yes

No

$p$ (Mantel-Cox)

Invasive multifocality

$$
\text { Yes }
$$

$6.5 \pm 0.3$

$6.7 \pm 0.1$

$=0.94$

$p$ (Mantel-Cox)

\section{Tumour burden}

$$
\begin{gathered}
\leq 19 \mathrm{~mm} \\
>19 \mathrm{~mm} \\
p(\text { Mantel-Cox })
\end{gathered}
$$

$6.5 \pm 0.1$

$=0.09$

\section{Tumour burden}

$6.7 \pm 0.1$
$7.1 \pm 0.1$

$=0.002$

$7.2 \pm 0.1$

$6.9 \pm 0.1$

$=0.01$

$7.1 \pm 0.1$

$6.9 \pm 0.2$

$7.1 \pm 0.1$

$=0.70$

$7.0 \pm 0.1$

$7.1 \pm 0.1$

$=0.59$

$7.1 \pm 0.1$

$6.9 \pm 0.1$

$=0.70$

$7.1 \pm 0.1$

$6.9 \pm 0.1$

$=0.03$

$<40 \mathrm{~mm}$

$6.7 \pm 0.1$

$7.1 \pm 0.1$ 

$\geq 40 \mathrm{~mm}$
$5.9 \pm 0.3$
$6.5 \pm 0.2$
$p$ (Mantel-Cox)
$=0.04$
$=0.02$

*All cases are censored

Table 7 The effects of selected variables on disease outcome (median RFS and OS) among the cases participating in the survival analysis 


\begin{tabular}{|c|c|c|c|c|}
\hline Variable & $\begin{array}{l}\text { Univariate } \\
\text { HR }(95 \% \mathrm{CI})\end{array}$ & $\mathbf{p}$ & $\begin{array}{l}\text { Multivariate } \\
\text { HR }(95 \% \text { CI })\end{array}$ & $\mathbf{p}$ \\
\hline $\begin{array}{l}\mathrm{pT} \geq 15 \text { vs. }<15 \\
\mathrm{~mm}\end{array}$ & $3.4(1.8-6.5)$ & $<0.001$ & $2.0(1.0-4.0)$ & 0.05 \\
\hline $\begin{array}{l}\text { Lymph node- } \\
\text { positive vs. lymph } \\
\text { node-negative }\end{array}$ & $3.8(2.3-6.4)$ & $<0.001$ & $3.0(1.7-5.3)$ & $<0.001$ \\
\hline $\begin{array}{l}\text { Grade } 2-3 \text { vs. } \\
\text { grade } 1 \text { (invasive } \\
\text { component) }\end{array}$ & $1.9(0.8-4.7)$ & 0.17 & & \\
\hline Presence of LVI & $1.8(1.1-2.9)$ & 0.02 & $1.1(0.6-1.7)$ & 0.97 \\
\hline HER2 positivity & $2.9(1.7-5.3)$ & $<0.001$ & $3.1(1.7-5.8)$ & $<0.001$ \\
\hline Triple negativity & $1.8(0.9-3.4)$ & 0.06 & $2.2(1.1-4.4)$ & 0.02 \\
\hline $\begin{array}{l}\text { Non-screen- } \\
\text { detected or } \\
\text { interval vs. screen- } \\
\text { detected }\end{array}$ & $2.5(1.4-4.3)$ & $<0.001$ & $1.6(0.9-2.8)$ & 0.15 \\
\hline $\begin{array}{l}\text { Casting } \\
\text { calcification vs. } \\
\text { spiculated tumour } \\
\text { mass }\end{array}$ & $1.5(0.6-3.8)$ & 0.37 & & \\
\hline $\begin{array}{l}\text { Spiculated tumour } \\
\text { mass vs. other }\end{array}$ & $1.1(0.7-1.9)$ & 0.69 & & \\
\hline $\begin{array}{l}\text { Presence of } \\
\text { multifocality }\end{array}$ & $0.8(0.5-1.4)$ & 0.45 & & \\
\hline $\begin{array}{l}\text { Tumour burden } \\
>19 \mathrm{~mm}\end{array}$ & $1.5(0.9-2.6)$ & 0.09 & & \\
\hline $\begin{array}{l}\text { Tumour burden } \\
>40 \mathrm{~mm}\end{array}$ & $1.8(1.1-3.4)$ & 0.05 & $1.0(0.5-2.0)$ & 0.98 \\
\hline
\end{tabular}

Table 8 The effects of selected patient- and tumour-related features on the risk of relapse according to the Cox proportional hazards model: univariate and multivariate analysis 


\begin{tabular}{|c|c|c|c|c|}
\hline Category & $\begin{array}{l}\text { Univariate } \\
\text { HR }(95 \% \mathrm{CI})\end{array}$ & $\mathbf{p}$ & $\begin{array}{l}\text { Multivariate } \\
\text { HR }(95 \% \mathrm{CI})\end{array}$ & $\mathbf{p}$ \\
\hline pT $\geq 15$ vs. $<15 \mathrm{~mm}$ & $8.9(2.1-37.7)$ & 0.03 & $2.5(1.2-5.2)$ & 0.03 \\
\hline $\begin{array}{l}\text { Lymph node } \\
\text { positivity }\end{array}$ & $5.0(2.1-11.9)$ & $<0.001$ & $3.3(1.4-8.2)$ & 0.01 \\
\hline $\begin{array}{l}\text { Grade } 2-3 \text { vs. grade } \\
1 \text { (invasive } \\
\text { component) }\end{array}$ & $25.2(0.2-3012.88)$ & 0.18 & & \\
\hline Presence of LVI & $2.9(1.3-6.1)$ & 0.01 & $1.3(0.6-3.0)$ & 0.48 \\
\hline HER2 positivity & $5.8(2.6-12.7)$ & $<0.001$ & $8.4(3.2-22.1)$ & $<0.001$ \\
\hline Triple negativity & $3.5(1.5-8.1)$ & 0.003 & $6.8(2.5-18.5)$ & $<0.001$ \\
\hline $\begin{array}{l}\text { Non-screen-detected } \\
\text { or interval vs. } \\
\text { screen-detected }\end{array}$ & $3.7(1.4-9.6)$ & 0.01 & $2.3(0.8-6.9)$ & 0.13 \\
\hline $\begin{array}{l}\text { Casting calcification } \\
\text { vs. } \\
\text { tumour mass }\end{array}$ & $1.7(0.5-6.4)$ & 0.42 & & \\
\hline $\begin{array}{l}\text { Spiculated tumour } \\
\text { mass vs. other }\end{array}$ & $1.3(0.5-3.0)$ & 0.59 & & \\
\hline $\begin{array}{l}\text { Presence of } \\
\text { multifocality }\end{array}$ & $0.8(0.3-1.9)$ & 0.59 & & \\
\hline $\begin{array}{l}\text { Tumour burden }>19 \\
\text { mm }\end{array}$ & $2.6(1.1 .-6.1)$ & 0.03 & $0.8(0.3-2.1)$ & 0.65 \\
\hline $\begin{array}{l}\text { Tumour burden }>40 \\
\text { mm }\end{array}$ & $2.6(1.1-6.2)$ & 0.03 & $0.9(0.3-2.7)$ & 0.94 \\
\hline
\end{tabular}

Table 9 The effects of selected patient- and tumour-related features on the risk of death due to breast cancer according to the Cox proportional hazards model: univariate and multivariate analysis 
4.3 The effect of the mammographic appearance on survival in patients with high risk breast cancer

After a median follow-up time of 78.5 (64.3-100.0) months, 29 patients (52.7\%) were free of relapse, 34 patients $(61.8 \%)$ were free of distant metastases, and $36(65.5 \%)$ patients survived. The median times of RFS, distant disease-free survival (DDFS) and OS were not yet reached at 100.0 months. Two patients developed local relapse, 3 contralateral breast cancers and 21 distant metastases as follows: 3 lung, 3 bone, 2 liver, 1 brain and 1 pleural relapse; in 7, 3 and 1 cases metastases affected 2, 3 and 4 organs at the same time. Two other patients developed second primary mesopharynx or colon cancer not rated as relapse.

The impact of the mammographic image on survival

The significant prognostic impact of the presence of casting-type calcifications on the mammogram was still observed. Survival data, according to whether the tumour was or was not associated with casting calcifications, are presented in table 10. 


\begin{tabular}{|c|c|c|c|c|c|}
\hline $\begin{array}{l}\text { Casting } \\
\text { calcifications }\end{array}$ & $\begin{array}{l}\text { Relapse- } \\
\text { free, } n\end{array}$ & $\begin{array}{l}\text { Surviving } \\
\mathrm{n}\end{array}$ & $\begin{array}{l}\text { Median RFS } \\
\text { months }\end{array}$ & $\begin{array}{l}\text { Median DDFS } \\
\text { months }\end{array}$ & $\begin{array}{l}\text { Median OS } \\
\text { months }\end{array}$ \\
\hline Present & $1 / 12$ & $6 / 12$ & 11.5 & 11.5 & 29.6 \\
\hline Absent & $28 / 43$ & $30 / 43$ & $>100$ & $>100$ & $>100$ \\
\hline $\mathbf{p}$ & 0.01 & 0.176 & $<0.001$ & $<0.001$ & 0.035 \\
\hline
\end{tabular}

Table 10 Outcome according to the presence or absence of casting calcifications on the mammogram 


\subsection{Cosmetic outcome 1-5 years after breast conservative surgery, irradiation and}

systemic therapy

A total of 198 patients were enrolled in the study. The mean age of the population was $62.0 \pm 10.6$ (range 25-89) years. The median follow-up time was 2.4 (range 1.2-5.9) years. Most of the tumours measured $\leq 2.0 \mathrm{~cm}$ and were lymph node-negative (Table 11). Data concerning the radiotherapy are presented in Table 12 . One hundred and sixty-seven patients $(84.3 \%)$ received only breast irradiation, while 31 patients $(15.7 \%)$ both breast and regional lymph node irradiation. Twenty patients (10.1\%) were treated with taxane-based chemotherapy before the radiotherapy. The systemic therapy before the radiotherapy started with an aromatase inhibitor or tamoxifen alone, in $49(24.7 \%)$ and 48 (24.2\%) cases, respectively. Four $(2.0 \%)$ and two $(1.0 \%)$ patients received a taxane-based chemotherapy and an aromatase inhibitor or tamoxifen thereafter, respectively. Seventy-five patients (37.9\%) did not participate in systemic therapy.

\section{Factors influencing cosmetic outcome}

The patients and the physicians considered the cosmetic outcome to be excellent or good in $76.3 \%$ and $47 \%$ of the cases, respectively; a weak correlation was observed between the opinions of the physicians and the patients (Table 13).

A large majority of the patients $(n=160,80.8 \%)$ underwent their breast surgery at our institute, and $127(84.1 \%)$ of them regarded the cosmetic outcome as excellent or good more often than did those who were operated on in smaller surgical departments $(n=24 / 38,63.2 \%)$ $(\mathrm{p}=0.05)$. In the view of the physicians, the cosmetic outcome overall was less often excellent or good as the tumour size increased: the mean $\pm \mathrm{SD}$ tumour size was $1.3 \pm 0.7$ and $1.5 \pm 0.7 \mathrm{~cm}$ in the excellent and good vs. the fair and poor outcome groups, respectively $(p=0.015)$. 
Among those patients who had an ABD, the physician considered the cosmetic outcome excellent or good in 29 cases $(37.7 \%)$, and fair or poor in 48 cases $(62.3 \%, p=0.04)$. A significant relation or interaction was not detected between these variables in the logistic regression analysis. The incidence and severity of hyperpigmentation, fibrosis, oedema and teleangiectasia are presented in Table 14. 


\begin{tabular}{|c|c|c|c|c|c|c|}
\hline \multirow[t]{2}{*}{$\begin{array}{l}\text { Patient and tumour } \\
\text { characteristics }\end{array}$} & \multirow[t]{2}{*}{$\begin{array}{c}\text { All } \\
\mathrm{n}(\%)\end{array}$} & \multicolumn{5}{|c|}{$\begin{array}{c}\text { Systemic therapy } \\
\mathrm{n}\end{array}$} \\
\hline & & Chemotherapy & Tamoxifen & $\begin{array}{l}\text { Aromatase } \\
\text { inhibitor }\end{array}$ & $\begin{array}{l}\text { Chemotherapy and } \\
\text { hormonal therapy }\end{array}$ & None \\
\hline \multicolumn{7}{|l|}{ Menostatus } \\
\hline Premenopausal & $48(24.2)$ & 12 & 22 & 0 & 4 & 10 \\
\hline Postmenopausal & $150(75.8)$ & 8 & 26 & 49 & 2 & 65 \\
\hline \multicolumn{7}{|l|}{ Age } \\
\hline$\leq 50$ years & $20(10.1)$ & 9 & 6 & 0 & 1 & 4 \\
\hline$>50$ years & $178(89.9)$ & 11 & 42 & 49 & 5 & 71 \\
\hline \multicolumn{7}{|l|}{ Lymph node surgery* } \\
\hline $\begin{array}{l}\text { Sentinel node biopsy } \\
\text { only }\end{array}$ & $111(56.0)$ & 6 & 28 & 29 & 3 & 45 \\
\hline $\begin{array}{l}\text { Axillary block } \\
\text { dissection }\end{array}$ & 77 (38.9) & 14 & 18 & 18 & 3 & 24 \\
\hline \multicolumn{7}{|l|}{ Tumour size } \\
\hline$\leq 2.0 \mathrm{~cm}$ & $164(82.8)$ & 12 & 41 & 41 & 3 & 67 \\
\hline$>2.0 \mathrm{~cm}$ & $34(17.2)$ & 8 & 8 & 7 & 3 & 8 \\
\hline
\end{tabular}




\section{Lymph node positivity}

Lymph node-negative

Lymph node-positive

$\begin{array}{ll}155(78.3) & 8 \\ 43(21.7) & 12\end{array}$

38

38

1

70

Tumour location

10

11

5

Upper quadrants

$\begin{array}{ll}130(65.6) & 16 \\ 34(17.2) & 1 \\ 34(17.2) & 3\end{array}$

\section{Central}

Lower quadrants
31

7

10

23

25
33

11

5

$125(63.1) \quad 12$

Current or

previous

$73(36.9) \quad 8$

smoker

* Ten patients did not participate in lymph node surgery because of their advanced age

Table 11 Patient and tumour characteristics in the overall study population and according to the systemic therapy 


\begin{tabular}{|c|c|c|c|c|c|c|}
\hline \multirow{2}{*}{$\begin{array}{l}\text { Radiotherapy- } \\
\text { related data }\end{array}$} & \multirow{2}{*}{$\begin{array}{c}\text { All } \\
\text { mean } \pm \mathrm{SD} \\
(\text { range })\end{array}$} & \multicolumn{5}{|c|}{$\begin{array}{l}\text { Systemic therapy } \\
\text { mean } \pm \text { SD (range) }\end{array}$} \\
\hline & & Chemotherapy & Tamoxifen & $\begin{array}{l}\text { Aromatase } \\
\text { inhibitor }\end{array}$ & $\begin{array}{l}\text { Chemotherapy } \\
\text { and hormonal } \\
\text { therapy }\end{array}$ & None \\
\hline $\begin{array}{l}\text { Volume of the } \\
\text { irradiated } \\
\text { breast }\left(\mathrm{cm}^{3}\right)\end{array}$ & $\begin{array}{l}1113.8 \pm 479.8 \\
(218-3620)\end{array}$ & $\begin{array}{l}1093.9 \pm 479.8 \\
(404-2059)\end{array}$ & $\begin{array}{l}974.7 \pm 447.9 \\
(218-2217)\end{array}$ & $\begin{array}{l}1140.5 \pm 413.5 \\
(484-2358)\end{array}$ & $\begin{array}{l}882.3 \pm 292.8 \\
(547-1249)\end{array}$ & $\begin{array}{c}1209.3 \pm 531.9 \\
(425-3620)\end{array}$ \\
\hline $\begin{array}{l}\mathbf{V}_{\mathbf{9 5} \%-107 \%} \\
(\%)\end{array}$ & $\begin{array}{l}88.9 \pm 2.6 \\
(81-95)\end{array}$ & $\begin{array}{l}89.3 \pm 2.9 \\
(84-95)\end{array}$ & $\begin{array}{l}88.9 \pm 2.7 \\
(81-94)\end{array}$ & $\begin{array}{l}88.7 \pm 2.5 \\
(82-94)\end{array}$ & $\begin{array}{l}90.0 \pm 2.8 \\
(86-94)\end{array}$ & $\begin{array}{c}89.0 \pm 2.4 \\
(81-94)\end{array}$ \\
\hline $\begin{array}{l}\mathbf{V}_{>\mathbf{1 0 7} \%} \\
(\%)\end{array}$ & $\begin{array}{l}0.6 \pm 1.1 \\
(0-6.9)\end{array}$ & $\begin{array}{l}0.4 \pm 0.5 \\
(0-1.9)\end{array}$ & $\begin{array}{l}0.9 \pm 1.6 \\
(0-6.9)\end{array}$ & $\begin{array}{l}0.8 \pm 1.2 \\
(0-4.5)\end{array}$ & $\begin{array}{l}0.1 \pm 0.2 \\
(0-0.5)\end{array}$ & $\begin{array}{l}0.4 \pm 0.7 \\
(0-4.1)\end{array}$ \\
\hline $\begin{array}{l}\text { Boost volume } \\
\left(\mathrm{cm}^{3}\right)\end{array}$ & $\begin{array}{l}79.4 \pm 45.3 \\
(13-258)\end{array}$ & $\begin{array}{l}82.3 \pm 39.7(32- \\
183)\end{array}$ & $\begin{array}{l}73.7 \pm 43.2 \\
(22-212)\end{array}$ & $\begin{array}{l}72.6 \pm 37.7 \\
(22-199)\end{array}$ & $\begin{array}{l}69.2 \pm 36.5 \\
(32-118)\end{array}$ & $\begin{array}{c}88.4 \pm 53.6 \\
(32-183)\end{array}$ \\
\hline $\begin{array}{l}\text { Breast } \\
\text { separation } \\
(\mathrm{cm})\end{array}$ & $\begin{array}{l}21.7 \pm 2.7 \\
(15.1-30.2)\end{array}$ & $\begin{array}{l}21.6 \pm 2.6(17.9- \\
25.7)\end{array}$ & $\begin{array}{l}22.0 \pm 2.8 \\
(16.8-30.2)\end{array}$ & $\begin{array}{l}21.1 \pm 3.1 \\
(15.1-30.1)\end{array}$ & $\begin{array}{l}20.4 \pm 1.7 \\
(18.1-22.5)\end{array}$ & $\begin{array}{c}21.9 \pm 2.5 \\
(16.4-28.9)\end{array}$ \\
\hline & $\mathrm{n}(\%)$ & & & $\mathrm{n}$ & & \\
\hline
\end{tabular}




$\begin{array}{lllllll}\begin{array}{l}\text { Photon boost } \\ \begin{array}{l}\text { Electron } \\ \text { boost }\end{array}\end{array} & 63(39.1) & 9 & 14 & 16 & 2 & 22 \\ \begin{array}{l}10 \text { Gy boost } \\ \text { dose }\end{array} & 145(90.1) & 19 & 25 & 26 & 3 & 33 \\ \begin{array}{l}16 \text { Gy boost } \\ \text { dose }\end{array} & 16(9.9) & 1 & 35 & 37 & 4 & 50 \\ \end{array}$

Table 12 Radiotherapy-related data on the overall study population and according to the systemic therapy 


\begin{tabular}{lllll}
\hline Cosmetic outcome & Excellent & Good & Fair & Poor \\
\hline Patient's opinion $(\%)$ & $93(47.0)$ & $58(29.3)$ & $44(22.2)$ & $3(1.5)$ \\
Physician's opinion $(\%)$ & $32(16.2)$ & $61(30.8)$ & $68(34.3)$ & $37(18.7)$ \\
Kappa & 0.09 & $\mathrm{p}<0.05$ & &
\end{tabular}

Table 13 Overall cosmetic outcome in the study population as assessed by the patient and the physician. The patients' subjective appreciation and the cosmetic result as classified by the physician (the absence of any abnormality, or the slight, moderate or severe manifestation of breast asymmetry or changes of the skin or the breast contour) are indicated. 


\begin{tabular}{lllll}
\hline & Score 0 $(\%)$ & Score 1 $(\%)$ & Score 2 $(\%)$ & Score 3 $(\%)$ \\
\hline Hyperpigmentation & $127(64.1)$ & $56(28.3)$ & $11(5.6)$ & $4(2.0)$ \\
Fibrosis & $131(66.1)$ & $37(18.7)$ & $30(15.2)$ & $0(0.0)$ \\
Oedema & $175(88.4)$ & $14(7.1)$ & $8(4.0)$ & $1(0.5)$ \\
Teleangiectasia & $176(88.9)$ & $3(1.5)$ & $6(3)$ & $13(6.6)$
\end{tabular}

Table 14 Incidence and severity of radiogenic changes of the skin, subcutaneous tissue and breast parenchyma according to the modified scoring system of Johansen et al. [36, 41] 


\section{The effect of patient and tumour characteristics on cosmetic outcome}

Thirty-one patients (19.7\%) complained of pain in the operated breast, while $81(40.9 \%)$ reported tenderness. Breast tenderness occurred significantly more often among premenopausal women or patients $\leq 50$ years old (both $\mathrm{p}<0.05$ ). The average \pm SD age of the patients who complained or had no complaint of breast tenderness was $59.8 \pm 11.6$ and $63.6 \pm 9.6$ years, respectively $(\mathrm{p}<0.05)$. Breast fibrosis and/or oedema occurred in $67(33.9 \%)$ and $23(11.6 \%)$ patients, respectively. Skin hyperpigmentation, found in altogether 71 patients $(35.9 \%)$ occurred in $59(83.1 \%)$ of the patients $>50$ years old, and $12(16.9 \%)$ of the women $\leq 50$ years old, respectively $(\mathrm{p}<0.05)$, and its incidence decreased with the median time elapsed after radiotherapy (2.1 and 2.5 years in the presence and the absence of hyperpigmentation, respectively, $\mathrm{p}=0.02)$. Teleangiectasia developed in 22 patients $(11.1 \%)$.

The mean $\pm \mathrm{SD}$ tumour size was $1.6 \pm 0.7 \mathrm{~cm}$ if moderate dyspigmentation occurred, and $1.3 \pm 0.7 \mathrm{~cm}$ in the other cases $(\mathrm{p}<0.05)$. The average $\pm S D$ tumour size was $1.9 \pm 1.4 \mathrm{~cm}$ if breast marked oedema occurred, and $1.4 \pm 0.7 \mathrm{~cm}$ in the other cases $(\mathrm{p}<0.05)$. Breast oedema occurred in $15(65.2 \%)$ and $8(34.8 \%)$ among those patients who had or did not have ABD, respectively $(p=0.01)$. Breast oedema was related to dyspigmentation $(p=0.003)$, fibrosis $(p<0.001)$ and breast asymmetry $(p=0.032)$, whereas none of these abnormalities were associated with teleangiectasia.

\section{Changes in body image and clothing habits}

Thirty-three (16.7\%) patients mentioned changes in their clothing habits and $44(22.3 \%)$ had experienced a variation in their body image. Those patients who noticed body image changes were younger than those who did not (the mean \pm SD age was $57.6 \pm 10.1$ vs. $63.3 \pm 10.4$ years, respectively, $\mathrm{p}<0.005$ ). Eighty-six percent of the postmenopausal, and $74 \%$ of the $\leq 50$ years old women needed to change their clothing habits ( $p<0.05$ ), while this measure was $8.9 \%$ and $1.3 \%$ according to whether the patient received or did not receive systemic therapy, respectively $(\mathrm{p}<0.005)$. 
The length of the excisional scar and breast asymmetry

In most cases, the excisional scar was in the upper quadrants $(n=130,65.7 \%)$. The mean \pm SD length of the scar was $8.2 \pm 3.5$ (range, 3.0-28.0) $\mathrm{cm}$. An average \pm SD breast asymmetry $(\mathrm{n}=159)$ of $2.7 \pm 1.9$ (range, 1.0-15.0) $\mathrm{cm}$ was found in 159 of the 194 evaluable patients (nipple excision was performed in 4 patients). The average $\pm \mathrm{SD}$ extent of breast asymmetry was $2.4 \pm 2.2 \mathrm{~cm}$ vs. $1.5 \pm 0.9 \mathrm{~cm}$ when the tumour was located in the upper vs. the lower quadrants, respectively $(\mathrm{p}=0.05)$, and $2.1 \pm 1.7 \mathrm{~cm}$ vs. $2.9 \pm 2.9 \mathrm{~cm}$ when the tumour diameter was $\leq 2 \mathrm{~cm}$ vs. $>2 \mathrm{~cm}$, respectively $(\mathrm{p}<0.05)$. The length of the scar did not influence any attribute of the cosmetic outcome.

\section{Radiotherapy and its effects on the cosmetic outcome}

More severe dyspigmentation and breast oedema occurred in patients with larger PTVs $(\mathrm{p}<0.001)$. The risk of more severe dyspigmentation and breast oedema increased by $18 \%$ and $23 \%$, respectively, for every $100 \mathrm{~cm}^{3}$ increase in irradiated breast volume $(\mathrm{OR}=1.18,95 \% \mathrm{CI}$ : 1.07-1.31; OR=1.23, 95\% CI: 1.12-1.36). Breast oedema was more frequent with increasing BS $(\mathrm{p}<0.005)$, and was not related to nodal irradiation. The incidence of breast fibrosis was significantly higher with larger PTVs (mean \pm SD value of PTV, patients with breast fibrosis: $1221.5 \pm 571.8 \mathrm{~cm}^{3}$ and patients without fibrosis: $\left.1058.7 \pm 416.9 \mathrm{~cm}^{3}, \mathrm{p}<0.05\right)$. The risk of breast fibrosis increased by $7 \%$ for every $100 \mathrm{~cm}^{3}$ increase in irradiated breast volume $(\mathrm{OR}=1.07,95 \% \mathrm{CI}: 1.00-1.14)$. No association was found between any of the attributes of cosmesis and the dose inhomogeneity within the irradiated volume. The dose inhomogeneity was related to the volume of the irradiated breast $(p=0.037)$. The risk of $V>107 \% \geq 1 \%$ increased by $8 \%$ for every $100 \mathrm{~cm}^{3}$ increase in irradiated breast volume $(\mathrm{OR}=1.078,95 \% \mathrm{CI}$ : $1.003-1.158)$.

A higher boost volume favoured breast fibrosis and oedema $(\mathrm{p}<0.005$ and $\mathrm{p}<0.001$, respectively). The risk of breast oedema and breast fibrosis increased by $21 \%$ and $12 \%$, respectively, for every $10 \mathrm{~cm}^{3}$ increase in boost volume (OR=1.21, 95\% CI: $1.09-1.33$ and $\mathrm{OR}=1.12,95 \%$, CI: 1.03-1.12). Breast oedema and/or fibrosis was more frequent among those patients who received a photon boost than those who received electrons (breast oedema: 13/63 
vs. 4/98, $\mathrm{p}=0.001$ and breast fibrosis: $26 / 63$ vs. $26 / 98, \mathrm{p}=0.038$ ). No association was found between the administration of systemic therapy and cosmetic or functional outcome.

\section{Smoking habits and their effect on cosmetic outcome}

One hundred and twenty-five $(63.1 \%)$ patients had never smoked, 49 (24.7\%) had smoked previously and 24 patients $(12.1 \%)$ smoked during the radiotherapy. Skin dyspigmentation and teleangiectasia developed significantly less often among the patients who had never smoked (both $\mathrm{p}<0.05)$.

\section{Discussion}

Mode of detection, mammographic appearance and multifocality as potential new prognostic factors in early breast cancer

Our findings in both the smaller and the second, extended database, are in accordance with those studies which demonstrated that the prognostic factors are more favourable in screendetected than in interval or symptomatic cancers. In numerous studies tumours were obviously smaller, more probably lymph node-negative, [42-48] and better differentiated [44, 46-47] if screen-detected. Some groups have reported that cancers of a special histological type, such as lobular or tubular carcinomas, are relatively more prevalent among screendetected tumours. $[44,46]$ In contrast, consistent with our data, no difference in histological type was observed among the different groups in the study by Gill et al. [47] LVI was less frequently present in screen-detected than in symptomatic cancers in that study. [47] We also found that LVI was more prevalent in interval or symptomatic cancers than in screendetected cancers. It has been suggested previously that one indicator of the less aggressive biological behaviour of screen-detected cancers is their higher hormone receptor content and the less frequent expression of HER2. Whereas Gill et al. [47] and Klemi et al. [43] reported that more screen-detected than symptomatic cancers were ER-positive, Joensuu et al. [46] did not discern any difference in the expression of ER or HER2 between screen-detected and symptomatic cancers. Similarly, we did not observe any difference in the ER, PR or HER2 status of the tumours as a function of the mode of detection. 
Among screen-detected cancers, less radical surgical interventions were needed, and less frequent chemotherapy was utilized, as a consequence of the earlier stage of these cancers. However, the hormone therapy requirements did not differ between the groups as concerns the mode of detection. This is a consequence of the similar distributions of the hormone receptor-positive tumours in the different groups, and the frequent use of endocrine therapy even in the early tumours, with the aim of the prevention of distant metastases, local relapses or metachronous second breast cancers.

Interval cancers are detected from the symptoms in the interval between scheduled screening episodes. A failure to detect breast cancer during screening depends on the testing procedure, the interpretation by the radiologist, and the patient and tumour characteristics. A biennial screening interval, a younger age [49-52] and increased breast density [52] favour detection failure. More importantly, the nature of interval cancers may influence their detection. Interval cancers have been demonstrated to occur more often in younger women, and to exhibit a higher proliferation rate, a lower ER expression and higher HER2 expression [49-52]. In our series, the interval cancers were significantly different from the screendetected cancers, and similar to the symptomatic cancers, as regards the tumour size, the lymph node status, the presence of LVI and the grade, but the differences in histological type, and ER/PR or HER2 status did not reach the level of statistical significance. This latter inconsistency with the literature data may be explained by the relatively low number of cases in our study. We found that a significant proportion of the interval cancers were mammographically occult even at the time of diagnosis. These tumours belong among a special subtype of interval cancers known as occult cancers [53].

Besides the classical prognostic factors (pT, lymph node status, grade, the presence or absence of LVI, and the expressions of the hormone receptors and HER2), however, other specific indicators for a better identification of the high-risk cases are needed. The mammographic appearance of the cancer has recently been suggested as a prognostic factor $[2,1-16,54]$. The risk of relapse or mortality in high-risk breast cancer patients was earlier found to be about threefold if the tumour was associated with casting calcifications on the mammogram [16]. In contrast, a highly favourable prognosis was experienced in small breast cancers appearing as stellate lesions on the mammogram [2, 14]. We also analyzed the potential role of mammographic image as a prognostic factor in a large database with a 
relatively large proportion of very good prognosis and short follow-up time, and also in 55 high-risk breast cancer patients with a longer follow-up. The outcome of this latter analysis is consistent with the literature data, and points to the long-persisting extreme difference in prognosis between cases with or without casting calcifications on the mammogram. Our observations underline that casting-type calcifications are not merely a marker of early relapse, but a marker of a special biologic nature with very poor outcome.

The prognostic significance of multifocality and the tumour burden have been investigated by many authors, but the results are inconclusive due to the different nomenclature used $[1,18$ 23]. In general, multifocality is defined as the presence of two or more tumour foci separated by normal breast parenchyma. Some studies have attempted to analyze the association between the entire tumour burden and the outcome, by using the aggregate measure of the dimensions or volumes of the tumour foci $[20,22,58,59]$. These factors have been demonstrated as determinants of poorer characteristics and outcome [1, 18, 19, 21-24]. This is why we intended to study these tumour features for the characterisation of breast carcinomas. In our extended database of operated early breast cancer patients, we found, that although multifocal breast tumours (frequently screen-detected) are often smaller than unifocal breast tumours, the aggregate size and hence the load of the cancer are larger, indicating a higher risk of dissemination. The relatively high proportion of lymph node-positive cases in invasive multifocal cancers reflects their aggressive behaviour and advanced stage. Although our results do not support the role of multifocality as an independent predictor of a worse prognosis, they should warn against the consideration of only a single tumour focus rather than the whole extent of the disease if multiple cancer foci are present so as to avoid false judgement.

In our study, multifocality and "invasive multifocality" occurred within the ranges reported by other authors $[22,24,55]$. There are a number of reasons for the discrepancies between the findings. Some authors do not distinguish between multicentric (situated in different quadrants of the breast) and multifocal cancers [18], and include tumours with a single invasive focus and in situ components [24], whereas others regard tumours as multifocal only if more than one invasive focus is present $[1,19-22,24,56]$. The strength of our study is that we relied on data recorded in thorough examinations of large-format histopathology slides. Nonetheless the retrospective nature of the study is a disadvantage. 
The UICC/AJC TNM system is used as a prognostic tool, and the TNM stage has long served as the basis of therapy decision-making. A major flaw is that, in cases of multifocality, the $\mathrm{T}$ stage indicates the largest invasive focus of the disease, but ignores the effective tumour burden, which may be significant if multiple foci are present. Our study accords with the findings of others in that the TNM system in its current form is not suitable for these purposes in the population of multifocal breast cancers $[18,21,56,58]$. We found that, despite the tumour being smaller, lymph node positivity was more prevalent among cancers containing multiple invasive foci, and a larger aggregate tumour burden involved a poorer outcome. These results are in accordance with those $[18,20,22,24,58,59]$ indicating that multifocality is related to lymph node positivity. Moreover, Tot et al. found multifocal and diffuse lesion distribution to be an independent predictor of breast cancer-related fatality [24]. In fact, the diffuse distribution of the lesions is a rarely described phenomenon, and consequently its effect should rather be analysed prospectively [57]. We could not recover this type of tumour from the pathology records; such cases were probably classified as unifocal, which could play a role in the incongruence between the findings of Tot et al. and ourselves [24].

For the estimation of tumour extent, we calculated the tumour burden by summing the largest diameters of the tumour foci. This method provides merely an approximation; only exact measurement of the entire tumour extent, encompassing the whole of the affected part of the breast parenchyma, would furnish accurate information. For the estimation of tumour burden, different approaches have been utilized in the literature. For assessment of the tumour burden of multifocal cancers and the effect on survival, Rezo et al. used aggregate sizes and volumes of the tumour foci, calculated as though they were spherical [22]. Interestingly, all measures gave similar results: increasing tumour size predicted a poorer outcome after 60 months of follow-up. Others followed the same method as we did, using the combined diameters of the tumour foci $[20,56,58]$.

The effect of multifocality on prognosis is controversial. Similarly to our findings, Cabioglu et al. concluded that the presence of multiple invasive foci favoured lymph node positivity, but the 55 month-survival did not differ between multifocal and unifocal cases [20]. Yersuhalmi et al. analysed a dataset on more than 25000 cases, and found that multifocality carried a $17 \%$ extra risk of breast cancer-related death in stage I-III breast cancers [21]. In a matched-pair analysis of 288 breast cancer cases, Weissenbacher et al likewise showed that both the risk of relapse and that of death due to breast cancer were increased in multifocal 
cancers [18]. Boyages et al. demonstrated a better 10-year survival rate among unifocal breast cancer cases than among multifocal breast cancer cases, but this effect was restricted to tumours $>20 \mathrm{~mm}$ [56]. In a series of 574 breast cancer cases, Tot et al. observed a significantly poorer BCSS rate in multifocal cancers, irrespective of whether only invasive or invasive plus in situ multifocal cases were included [24]. We did not detect a difference in survival between the cases with multifocal and unifocal breast cancers, but tumour size, lymph node status and HER2-positive or triple negative status were independent predictors of outcome. The relatively low overall number of events and short follow-up times could have played a role in these results.

In Hungary, the national mammographic breast-screening program was introduced in 2001. The quality indicators of the screening closely match the European guidelines, with the exception of the participation rate, which is around $40 \%$. However, the proportion of women regularly screened for breast cancer is more than $60 \%$ as a result of the contribution of opportunistic screening. Thus, in this mixed population of early breast cancer patients, the use of tumour features such as the mode of detection and the mammographic appearance, the multifocality of the tumour represent useful tools for individualized care.

Evaluation of the cosmetic and functional outcomes after breast-conserving surgery and conformal radiotherapy, with or without adjuvant systemic therapy

The cosmetic and the functional outcome after the surgical and oncoradiological treatment play an important role in the breast cancer patients' quality of life. These factors depend on many patient- and therapy-related characteristics. The age, the menopausal status, the weight and the general health status of the patient, the stage of the tumour and the surgical intervention clearly influence the results [30]. The radiogenic changes of the breast depend on the irradiation's features and the individual radiosensitivity [30-33]. Adjuvant chemotherapy [41] and tamoxifen therapy $[41,76]$ have been suggested to predict a poor cosmetic outcome [38].

More than three-quarters of the patients considered the cosmetic outcome to be excellent or good, which is similar to the observations by other groups $[36,38,60]$. In contrast, in the opinion of the physicians, only about half of the cases belonged in this category. One explanation of the discrepancy might be the strict conditions used for the evaluation of the 
cosmetic appearance. A further contributing factor could have been the relatively short follow-up time in our study since the consideration of the radiation side-effects by the patient may change in time [37].

The location and stage of the tumour clearly determine the cosmetic outcome [36, 60-64]. In our study, breast oedema, fibrosis, teleangiectasia and dyspigmentation were all related to the size of the tumour, and were interrelated. Similarly as in the studies by Johansen et al. and Taylor et al., we found that tumours located in the upper quadrants and those with a larger diameter were predisposed to more severe breast asymmetry [36, 60].

The available data are not completely unequivocal as regards the relation between a young age and the cosmetic and functional outcomes. Most studies report an improved cosmetic outcome among younger women $[38,60,65,66]$ though the opposite to has been suggested [36]. We did not find any age-specific differences in the overall cosmetic outcome. However, the different components of the cosmetic and functional results did depend on the age of the patient. The more frequent breast tenderness or pain among $\leq 50$ years old women might have been related to hormonal effects and the change in body image in this age group could have been dependent on psychological or mental factors. The higher incidence of dyspigmentation in those over 50 years of age is probably due to the age-dependent response to radiation, with an increased accumulation of melanin and lipofuscin, the pigments related to aging and oxidative stress [67]. It is well known that radiogenic side-effects are more frequent for larger breasts. Nevertheless, to the best of our knowledge, this analysis is the first to report cosmetic results after conformal breast radiotherapy in relation to dose-volume data. Although conformal radiotherapy was applied in the study by Lilla et al., a detailed analysis of the radiotherapy-related data was not reported [66]. In that and other studies, the associations between the side-effects and the irradiated volume were based on approximate data such as the size of the breast or the bra cup [36, 41, 66, 68, 69] and the chest wall separation [68]. Likewise, dose homogeneity in the entire PTV was predicted after visualization of the dose distribution at the central axis $[64,68]$ or was related to the use or avoidance of tissue compensators or wedges $[60,70,71]$. In one of these studies, the dose homogeneity demonstrated a parallel with the breast size [68], an effect confirmed by our results. In our study, no association was detected between the dose inhomogeneity and poorer cosmesis as a result of restricting the overdosed volume (V>107\%) to $1 \%$ of the PTV, and the superposition 
of individually weighted 6 or $15 \mathrm{MV}$ segmental fields to the tangential fields [40]. Our finding that the photon boost was more often related to breast oedema and fibrosis is in accordance with the outcome of the robust analysis by Murphy et al. [69]. We consider that this phenomenon is a consequence of the larger volume irradiated when 2 photon fields are applied as compared with the use of one direct electron field. For the best cosmetic result, the use of an electron boost is recommended, or the intensity-modulated radiation therapy (IMRT) technique may be utilized [72-74].

Systemic therapy has been found detrimental to the cosmetic and functional outcome in many studies $[35,41,60,64,69,75]$. Most investigated the effects of chemotherapy with cyclophosphamide, methotrexate and fluorouracil (CMF) or an antracycline-containing regimen, either concurrently or sequentially with radiotherapy [36, 60, 75]. In the study by Johansen et al., chemotherapy with CMF or with tamoxifen was associated with a 5-fold (CMF) or 10-fold (tamoxifen) higher risk of breast fibrosis, respectively [36]. Taxane-based combinations have become a routine option in adjuvant chemotherapy, but the effects of sequentially applied taxane-based chemotherapy on breast cosmesis have not yet been reported. We did not detect adverse effects of such a regimen, though the number of patients was low. The third-generation aromatase inhibitors currently compromise the standard endocrine therapy of postmenopausal women with hormone-dependent breast cancer. However, tamoxifen is still administered in premenopausal and selected postmenopausal women. Azria et al. demonstrated in their primal work that letrozole does not exert a detrimental effect on early or late radiation skin toxicity [33]. In accordance with these findings, aromatase inhibitor therapy in our analysis did not have any effect on the studied parameters. In contrast, the prospective study by Azria et al. revealed that the concurrent administration of tamoxifen with radiotherapy doubled the risk of subcutaneous fibrosis [31]. Likewise, we found that concomitant tamoxifen increased the risk of lung fibrosis $(\mathrm{OR}=2.442)$ [40]. Due to the limitations of the analyses, other reports have not provided a clear answer as to the effect of simultaneous tamoxifen therapy with radiotherapy on the cosmetic outcome $[41,60,61,76]$. Our survey suggested that tamoxifen therapy was related to the change in body image, but since no specific radiogenic changes were detected, the frequent weight gain associated with this medication may have played a role in this outcome measure. 
The patient and tumour characteristics influence significantly the cosmetic outcome; therapyrelated factors can also modify the breast aesthetics and the patient well-being. Although most of the patients were satisfied with the cosmetic outcome, individual planning of the oncoradiological therapy has considerable importance of reaching the best cosmetic result.

\section{Summary, conclusions}

6.1 Our findings reveal that screen-detected cancers have a more favourable prognosis and need less oncological treatment than do tumours detected outside mammographic service screening. The mammographic appearance of a tumour reflects its biological behaviour, and should be considered when the management is to be optimized.

6.2 For the adequate management of breast cancer, an appropriate assessment of the tumour distribution is essential; heightened attention is needed during the care of multifocal breast cancers, which present in a more advanced stage than estimated from a he consideration of only the largest focus.

6.3 Our results gave further evidence of the poorer prognosis of those tumours which show casting-type calcification on the mammogram and highlight the importance of this special type of tumour features.

6.4 The cosmetic outcome after breast-conserving surgery is primarily determined by the stage of the disease and the consequences of radiotherapy. Despite the achievements made regarding the effectiveness and side-effect profile of modern radiotherapy, a careful estimate of its benefits remain necessary in each case and determination of the individual treatment strategy accordingly. 


\section{Acknowledgements}

I wish to express my special thanks to my supervisor Professor Zsuzsanna Kahán director of the Department of Oncotherapy, University of Szeged for her support and scientific guidance of my work.

I would like to thank Professor László Thurzó former director of the Department of Oncotherapy, University of Szeged, who provided excellent working conditions for me at the institute.

Special thanks are due to the staff members of the breast board: Professor György Lázár, Attila Paszt, Zsolt Simonka, Katalin Ormándi, Csilla Hoffmann, Sándor Hamar, László Kaizer, András Vörös, Erika Csörgő, Máté Lázár, without whom this task would never have been fulfilled.

Special thanks are also due to Tibor Nyári, József Eller and Zoltán Varga for their guidance in the statistical analysis.

I greatly appreciate all the support and work of high standard provided by physicians, technicians and physicists of the Department of Oncotherapy, University of Szeged that helped this dissertation to be born.

I would like to thank to my family and friends for encouraging and supporting me. 


\section{References}

1. Tot T. The metastatic capacity of multifocal breast carcinomas: extensive tumours versus tumours of limited extent. Hum Pathol 2009; 40: 199-205.

2. Tabar L, Chen THH, Yen AMF, Tot T, Tung TH et al. Mammographic tumour features can predict long-term outcomes reliably in women with 1-14-mm invasive breast carcinoma. Cancer 2004; 101: 1745-1759.

3. Tot T, Kahán Z. Springer, Dordrecht, Heidelberg, London, New York, 2011. pp. 1-22. A new approach to early breast cancer. In: Breast cancer, a heterogeneous disease entity. The very early stages.

4. Kahán Z. New approaches for the classification of early breast cancer, a heterogeneous disease entity. memo, 2011; 4: 135-139.

5. Majek O, Danes J, Skovajsova M, Bartonkova H, Buresova L et al. Breast cancer screening in the Czech Republic: time trends in performance indicators during the first seven years of the organised programme. BMC Public Health 2011; 11: 288.

6. Gill PG, Farshid G, Luke CG, Roder DM. Detection by screening mammography is a powerful independent predictor of survival in women diagnosed with breast cancer. Breast 2004; 13: 15-22.

7. Tabar L, Fagerberg G, Duffy SW et al. The Swedish two-county trial of mammographic screening for breast cancer: recent results and calculation of benefit. $\mathbf{J}$ Epidemiol Community Health 1989; 43:107-114

8. Tabar L, Yeng M-F, Vitak B et al. Mammography service screening and mortality in breast cancer patients: 20-year follow-up before and after introduction of screening. Lancet $2003 ; 361: 1405-1410$

9. The Swedish Organised Service Screening Evaluation Group Reduction of breast cancer mortality from organised service screening with mammography: 1. Further confirmation with extended data. Cancer Epidemiol Biomarkers Prev 2006; 15:45-51

10. Miller AB, Baines $\mathrm{C}$, To $\mathrm{T}$ et al. Canadian national breast screening study. 1. Breast cancer detection and death rates among women aged 40 to 49 years. 1992; CMAJ 147:14591476 
11. Kerlikowske K, Grady D, Rubin SM et al. Efficacy of screening mammography. A meta-analysis. JAMA 1995; 273:149-154

12. Tabar L, Dean PB, Kaufman CS et al. A new era in the diagnosis of breast cancer. Surg Oncol Clinics NA 2000; 9:233-277

13. Thurfjell E, Thurfjell MG, Lindgren A Mammographic finding as predictor of survival in 1-9 $\mathrm{mm}$ invasive breast cancers. Worse prognosis for cases presenting as calcifications alone. Breast Cancer Res Treat 2001; 67:177-180

14. Alexander MC, Yankaskas BC, Biesemier KW Association of stellate mammographic pattern with survival in small invasive breast tumours. Am J Roentgenol 2006; 187:29-73

15. Peacock C, Given-Wilson RM, Duffy SW Mammographic casting-type calcification associated with small screen-detected invasive breast cancers: is this a reliable prognostic indicator? Clin Radiol 2004; 59:165-170

16. Pálka I, Ormándi K, Gaál $\mathrm{S}$ et al. Casting-type calcifications on the mammogram indicate a higher probability of early relapse and death among operable high-risk breast cancer patients. Acta Oncol 2007; 46(8):1178-83.

17. Alexander MC, Yankaskas BC, Biesemier KW Association of stellate mammographic pattern with survival in small invasive breast tumours. Am J Roentgenol 2006; 187:29-73

18. Weissenbacher TM, Zschage M, Janni W, Jeschke U, Dimpfl T et al. Multicentric and multifocal versus unifocal breast cancer: is the tumour-node-metastasis classification justified? Breast Cancer Res Treat 2010; 122: 27-34.

19. Tot T, Pekar G, Hofmeyer S, Sollie T, Gere M, Tarjan M. The distribution of lesions in 1-14-mm invasive breast carcinomas and its relation to metastatic potential. Virchows Arch 2009; 455: 109-115.

20. Cabioglu N, Ozmen V, Kaya H, Tuzlali S, Igci A et al. Increased lymph node positivity in multifocal and multicentric breast cancer. J Am Coll Surg 2009; 208: 67-74.

21. Yerushalmi R, Kennecke H, Woods R, Olivotto IA, Speers $\mathrm{C}$ et al. Does multicentric/multifocal breast cancer differ from unifocal breast cancer? An analysis of survival and contralateral breast cancer incidence. Breast Cancer Res Treat 2009; 117: 365 370.

22. Rezo A, Dahlstrom J, Shadbolt B, Rodins K, Zhang Y et al. Tumour size and survival in multicentric and multifocal breast cancer. Breast 2011; 20: 259-263. 
23. Kurtz JM, Jacquemier J, Amalric R, Brandone H, Ayme Y et al. Breast-conserving therapy for macroscopically multiple cancers. Ann Surg 1990; 212: 38-44.

24. Tot T, Gere M, Pekar G, Tarjeb M, Hofmeyer S et al. Breast cancer multifocality, disease extent, and survival. Hum Pathol 2011; 42: 1761-9

25. Voogd AC, Nielsen M, Peterse JL, Blichert-Toft M, Bartelink H, Overgaard M, et al. Differences in risk factors for local and distant recurrence after breast-conserving therapy or mastectomy for stage I and II breast cancer: pooled results of two large European randomized trials. J Clin Oncol 2001; 19:1688-1697.

26. Fisher B, Anderson S, Bryant J, Margolese RG, Deutsch M, Fisher ER, et al. Twentyyear follow-up of a randomized trial comparing total mastectomy, lumpectomy, and lumpectomy plus irradiation for the treatment of invasive breast cancer. N Engl J Med 2002; 347:1233-1241.

27. Veronesi U, Cascinelli N, Mariani L, Greco M, Saccozzi R, Luini A, et al. Twentyyear follow-up of a randomized study comparing breast-conserving surgery with radical mastectomy for early breast cancer. N Engl J Med 2002; 347:1227-1232.

28. Offersen BV, Overgaard M, Kroman N, Overgaard J. Accelerated partial breast irradiation as part of breast conserving therapy of early breast carcinoma: a systematic review. Radiother Oncol 2009; 90:1-13.

29. Fyles AW, McCready DR, Manchul LA, Trudeau ME, Merante P, Pintilie M, et al. Tamoxifen with or without breast irradiation in women 50 years of age or older with early breast cancer. N Engl J Med 2004; 351:963-970.

30. Munshi A, Kakkar S, Bhutani R, Jalali R, Budrukkar A, Dinshaw KA. Factors influencing cosmetic outcome in breast conservation. Clin Oncol (R Coll Radiol) 2009; 21:285-293.

31. Azria D, Gourgou S, Sozzi WJ, Zouhair A, Mirimanoff RO, Kramar A, et al. Concomitant use of tamoxifen with radiotherapy enhances subcutaneous breast fibrosis in hypersensitive patients. Br J Cancer 2004; 91:1251-1260.

32. Azria D, Betz M, Bourgier C, Sozzi WJ, Ozsahin M. Identifying patients at risk for late radiation-induced toxicity. Crit Rev Oncol Hematol, 2010; Epub ahead of print.

33. Azria D, Belkacemi Y, Romieu G, Gourgou S, Gutowski M, Zaman K, et al. Concurrent or sequential adjuvant letrozole and radiotherapy after conservative surgery for 
early-stage breast cancer (CO-HO-RT): a phase 2 randomised trial. Lancet Oncol 2010; $11: 258-265$.

34. Bartelink H, Horiot JC, Poortmans PM, Struikmans H, Van den Bogaert W, Fourquet A, et al. Impact of a higher radiation dose on local control and survival in breast-conserving therapy of early breast cancer: 10-year results of the randomized boost versus no boost EORTC 22881-10882 trial. J Clin Oncol 2007; 25:3259-3265.

35. Collette S, Collette L, Budiharto T, Horiot JC, Poortmans PM, Struikmans H, et al. Predictors of the risk of fibrosis at 10 years after breast conserving therapy for early breast cancer: a study based on the EORTC Trial 22881-10882 'boost versus no boost'. Eur J Cancer 2008; 44:2587-2599.

36. Johansen J, Overgaard J, Rose C, Engelholm SA, Gadeberg CC, Kjaer M, et al. Cosmetic outcome and breast morbidity in breast-conserving treatment--results from the Danish DBCG-82TM national randomized trial in breast cancer. Acta Oncol 2002; 41:369380.

37. Curran D, van Dongen JP, Aaronson NK, Kiebert G, Fentiman IS, Mignolet F, et al. Quality of life of early-stage breast cancer patients treated with radical mastectomy or breast-conserving procedures: results of EORTC Trial 10801. The European Organization for Research and Treatment of Cancer (EORTC), Breast Cancer Co-operative Group (BCCG). Eur J Cancer 1998; 34:307-314.

38. Cardoso MJ, Cardoso J, Santos AC, Vrieling C, Christie D, Liljegren G, et al. Factors determining esthetic outcome after breast cancer conservative treatment. Breast J 2007; 13:140-146.

39. Nikolenyi A, Sukosd F, Kaizer L, Csorgo E, Voros A et al. Tumour topoisomerase II alpha status and response to anthracycline-based neoadjuvant chemotherapy in breast cancer. Oncology 2011; 80: 269-277

40. Kahan Z, Csenki M, Varga Z, Szil E, Cserhati A, Balogh A, et al. The risk of early and late lung sequelae after conformal radiotherapy in breast cancer patients. Int J Radiat Oncol Biol Phys 2007; 68:673-681.

41. Johansen J, Overgaard J, Overgaard M. Effect of adjuvant systemic treatment on cosmetic outcome and late normal-tissue reactions after breast conservation. Acta Oncol 2007; 46:525-533. 
42. Moody-Ayers SY, Wells CK, Feinstein AR "Benign" tumours and "early detection" in mammography-screened patients of a natural cohort with breast cancer. Arch Intern Med 2000; 160:1109-1115

43. Klemi PJ, Joensuu H, Tikkanen S et al. Aggressiveness of breast cancers found with and without screening. Br Med J 1992; 304:467-469

44. Klemi PJ, Parvinen I, Pylkkanen L et al. Significant improvement in breast cancer survival through population-based mammography screening. Breast 2003; 12:308-313

45. Anttinen J, Kautiainen H, Kuopo T Role of mammography screening as a predictor of survival in postmenopausal breast cancer patients. Br J Cancer 2006; 94:147-151

46. Joensuu H, Lehtimäki $\mathrm{T}$, Holli $\mathrm{K}$ et al. Risk for distant recurrence of breast cancer detected by mammography screening or other methods. JAMA 2004; 292:1064-1073

47. Gill PG, Farshid G, Luke CG et al. Detection by screening mammography is a powerful independent predictor of survival in women diagnosed with breast cancer. Breast $2005 ; 13: 15-22$

48. Ernst MF, Voogd AC, Goebergh JWW et al. Breast carcinoma diagnosis, treatment, and prognosis before and after the introduction of mass mammographic screening. Cancer 2004; 100:1337-1344

49. Porter PJ, El-Bastawissi AZ, Mandelson MT et al. Breast tumour characteristics as predictors of mammographic detection: Comparison of interval- and screen-detected cancers. J Natl Cancer Inst 1999; 91:2020-2028

50. Gilliland FD, Joste N, Stauber PM et al. Biologic characteristics of interval and screen-detected breast cancers. J Natl Cancer Inst 2000; 92:743-749

51. Crosier M, Scott D, Wilson RG et al. Differences in Ki67 and c-erbB2 expression between screen-detected and true interval breast cancers. Clin Cancer Res 1999; 5:26822688

52. Porter GJ, Evans AJ, Cornford EJ et al. Influence of mammographic parenchymal pattern in screen-detected and interval invasive breast cancers on pathologic features, mammographic features, and patient survival. Am J Roentgenol 2007; 188:676683

53. Porter GJ, Evans AJ, Burrell HC et al. NHSBSP type 1 interval cancers: a scientifically valid grouping? Clin Radiol 2007; 62:262-267 
54. Zunzunegui RG, Chung MA, Oruwari J et al. Casting-type calcifications with invasion and high-grade ductal carcinoma in situ. Arch Surg 2003; 138:537-540

55. Bendifallah S, Werkoff G, Borie-Moutafoff C, Antoine M, Chopier J et al. Multiple synchronous (multifocal and multicentric) breast cancer: clinical implications. Surg Oncol 2010; 19: 115-23.

56. Boyages J, Jayasinghe UW, Coombs N. Multifocal breast cancer and survival: each focus does matter particularly for larger tumours. Eur J Cancer 2010; 46: 1990-1996.

57. Tot T. The diffuse type of invasive lobular carcinoma of the breast: morphology and prognosis. Virchows Arch 2003; 443: 718-724.

58. Andea AA, Bouwman D, Wallis T, Visscher DW. Correlation of tumour volume and surface area with lymph node status in patients with multifocal/multicentric breast carcinoma. Cancer 2004; 100: 20-27.

59. Coombs NJ, Boyages J. Multifocal and multicentric breast cancer: does each focus matter? J Clin Oncol 2005; 23: 7497-7502.

60. Taylor ME, Perez CA, Halverson KJ, Kuske RR, Philpott GW, Garcia DM, et al. Factors influencing cosmetic results after conservation therapy for breast cancer. Int J Radiat Oncol Biol Phys 1995; 31:753-764.

61. Wazer DE, Morr J, Erban JK, Schmid CH, Ruthazer R, Schmidt-Ullrich RK. The effects of postradiation treatment with tamoxifen on local control and cosmetic outcome in the conservatively treated breast. Cancer 1997; 80:732-740.

62. Van Limbergen E, Rijnders A, van der Schueren E, Lerut T, Christiaens R. Cosmetic evaluation of breast conserving treatment for mammary cancer. 2. A quantitative analysis of the influence of radiation dose, fractionation schedules and surgical treatment techniques on cosmetic results. Radiother Oncol 1989; 16:253-267.

63. Borger JH, Kemperman H, Smitt HS, Hart A, van Dongen J, Lebesque J, et al. Dose and volume effects on fibrosis after breast conservation therapy. Int $\mathbf{J}$ Radiat Oncol Biol Phys 1994; 30:1073-1081.

64. Wazer DE, DiPetrillo T, Schmidt-Ullrich R, Weld L, Smith TJ, Marchant DJ, et al. Factors influencing cosmetic outcome and complication risk after conservative surgery and radiotherapy for early-stage breast carcinoma. J Clin Oncol 1992; 10:356-363.

65. Pezner RD, Patterson MP, Hill LR, Vora NL, Desai KR, Lipsett JA. Breast retraction assessment. Multiple variable analysis of factors responsible for cosmetic retraction in 
patients treated conservatively for stage I or II breast carcinoma. Acta Radiol Oncol 1985; 24:327-330.

66. Lilla C, Ambrosone CB, Kropp S, Helmbold I, Schmezer P, von Fournier D, et al. Predictive factors for late normal tissue complications following radiotherapy for breast cancer. Breast Cancer Res Treat 2007; 106:143-150.

67. Porta EA. Pigments in aging: an overview. Ann N Y Acad Sci 2002; 959:57-65.

68. Moody AM, Mayles WP, Bliss JM, A'Hern RP, Owen JR, Regan J, et al. The influence of breast size on late radiation effects and association with radiotherapy dose inhomogeneity. Radiother Oncol 1994; 33:106-112.

69. Murphy C, Anderson PR, Li T, Bleicher RJ, Sigurdson ER, Goldstein LJ, et al. Impact of the radiation boost on outcomes after breast-conserving surgery and radiation. Int $\mathbf{J}$ Radiat Oncol Biol Phys 2011 Sep 1;81(1):69-76.

70. Ellenbogen R, Jankauskas S, Collini FJ. Achieving standardized photographs in aesthetic surgery. Plast Reconstr Surg 1990; 86:955-961.

71. Sarin R, Dinshaw KA, Shrivastava SK, Sharma V, Deore SM. Therapeutic factors influencing the cosmetic outcome and late complications in the conservative management of early breast cancer. Int J Radiat Oncol Biol Phys 1993; 27:285-292.

72. Hill-Kayser CE, Chacko D, Hwang WT, Vapiwala N, Solin LJ. Long-term clinical and cosmetic outcomes after breast conservation treatment for women with early-stage breast carcinoma according to the type of breast boost. Int J Radiat Oncol Biol Phys, 2011; Mar $15 ; 79(4): 1048-54$.

73. Harsolia A, Kestin L, Grills I, Wallace M, Jolly S, Jones C, et al. Intensity-modulated radiotherapy results in significant decrease in clinical toxicities compared with conventional wedge-based breast radiotherapy. Int J Radiat Oncol Biol Phys 2007; 68:1375-1380.

74. McDonald MW, Godette KD, Whitaker DJ, Davis LW, Johnstone PA. Three-year outcomes of breast intensity-modulated radiation therapy with simultaneous integrated boost. Int J Radiat Oncol Biol Phys 2010; 77:523-530.

75. Abner AL, Recht A, Vicini FA, Silver B, Hayes D, Come S, et al. Cosmetic results after surgery, chemotherapy, and radiation therapy for early breast cancer. Int $\mathbf{J}$ Radiat Oncol Biol Phys 1991; 21:331-338. 
76. Fowble B, Fein DA, Hanlon AL, Eisenberg BL, Hoffman JP, Sigurdson ER, et al. The impact of tamoxifen on breast recurrence, cosmesis, complications, and survival in estrogen receptor-positive early-stage breast cancer. Int J Radiat Oncol Biol Phys 1996; 35:669-677. 
APPENDIX 